\title{
Basaltos continentais do Cretáceo Inferior da bacia de Campos, SE do Brasil: compilação de dados e petrogênese
}

\author{
Janaína Teixeira Lobo ${ }^{1}$, Beatriz Paschoal Duarte ${ }^{2}$, Peter Szatmari ${ }^{3}$ \& Sérgio de Castro Valente ${ }^{4}$
}

\begin{abstract}
Resumo Este trabalho apresenta dados geoquímicos e discute modelos petrogenéticos em base quantitativa do processo de evolução em câmara magmática dos basaltos de Campos. Dados geoquímicos foram selecionados da literatura (Fodor \& Vetter 1984 e Mizusaki et al. 1992). A compilação destes dados obedeceu aos seguintes critérios: (1) soma dos óxidos no intervalo de 99 - 101\% em peso, (2) baixos valores de perda ao fogo e, (3) norma CIPW para a caracterização dos toleítos (apresentação de hiperstênio normativo em todas as amostras). Através de modelamento geoquímico quantitativo e métodos estatísticos foram descartados os processos de cristalização fracionada, mistura magmática e assimilação simples para a geração dos basaltos de Campos. Os dados do modelo proposto, suportados pelos dados isotópicos, apontaram para o processo evolutivo de assimilação concomitante a cristalização fracionada (AFC). Os basaltos do Cretáceo Inferior da bacia de Campos evoluíram a partir de cerca de $30 \%$ de AFC, envolvendo cristalização fracionada de uma assembléia composta essencialmente por $5 \%$ de olivina, $30 \%$ de clinopiroxênio e $65 \%$ de plagioclásio em câmara magmática localizada na crosta inferior. O envolvimento de crosta inferior local no processo evolutivo dos basaltos de Campos encontra apoio na pronunciada anomalia negativa de $\mathrm{Nb}\left(\mathrm{La} / \mathrm{Nb}_{\mathrm{N}}\right.$ até 5,30$)$ detectada principalmente nos seus membros mais evoluídos.
\end{abstract}

Palavras-chave: basalto, modelamento geoquímico, processo evolutivo.

\begin{abstract}
Continental within-plate Lower Cretaceous basalts in the Campos basin, SE Brazil: compilation of data and petrogenisis. This paper presents geochemical data and discusses, on a quantitative basis, petrogenetic models of the process by which the basalts of the Campos basin, SE Brazil, evolved in their magma chamber. Geochemical data were selected from the literature (Fodor \& Vetter 1984 e Mizusaki et al. 1992). In the compilation of these data we observed the following criteria: (1) sum of oxides are in the 99 $101 \mathrm{wt. \%}$ interval; (2) ignition losses are low; (3) CIPW norm is in the tholeiitic range (hypersthene normative for all samples). Quantitative geochemical modeling and statistical methods does not allow us to derive the Campos basalts by the solitary activity of a single process such as fractional crystallization, magma mixing, or simple assimilation. Rather, the proposed model, supported by isotopic data, points to an evolutionary process consisting of assimilation concomitant with fractional crystallization $(A F C)$. The early Cretaceous basalts of the Campos basin evolved by about $30 \% A F C$, involving crystallization of an assembly made up essentially of $5 \%$ of olivine, $30 \%$ of clinopyroxene, and $65 \%$ of plagioclase in a magma chamber contained within the lower crust. Local involvement of the lower crust in the evolutionary process of the Campos basalts is supported by the pronounced negative anomaly of $\mathrm{Nb}\left(\mathrm{La} / \mathrm{Nb}_{\mathrm{N}}\right.$ up to 5.30) detected mainly in the most evolved members.
\end{abstract}

Keyworks: basalt, geochemical modelling, evolution process

INTRODUÇÃO A bacia de Campos situa-se na costa norte do Estado do Rio de Janeiro e sul do Estado do Espírito Santo, entre os paralelos $21^{\circ}$ e $23^{\circ} \mathrm{S}$ (Fig. 1). Esta bacia teve sua origem relacionada à fragmentação do supercontinente Gondwana e posterior evolução do rifte do Oceano Atlântico Sul a partir do Cretáceo Inferior. A seqüência estratigráfica da bacia de Campos inclui derrames basálticos, predominantemente toleíticos (basaltos de Campos), de até $600 \mathrm{~m}$ de espessura, e que cobriram uma área estimada em $100.000 \mathrm{Km}^{2} \mathrm{e}$ volume aproximado de $60.000 \mathrm{Km}^{3}$ (Mizusaki et al. 1988).

Este trabalho apresenta dados geoquímicos e discute modelos petrogenéticos em base quantitativa do processo de evolução do líquido magmático em câmara para a geração dos basaltos do Cretáceo Inferior da bacia de Campos, SE do Brasil.

TRABALHOS ANTERIORES Os primeiros trabalhos envolvendo os basaltos do Cretáceo Inferior da bacia de Campos foram sob o ponto de vista geocronológico onde idades de 124 até $112 \mathrm{Ma}$ foram encontradas (Fodor et al. 1983).

Posteriormente, os estudos englobaram análises litogeoquímicas de amostras basálticas, de idades entre

\footnotetext{
1 - Rede de Estudos em Geotectônica - PETROBRAS/CENPES/UERJ, Rio de Janeiro, RJ, Brasil. E-mail: janaina.lobo@uerj.br

2 - TEKTOS/FGEL/UERJ, Rio de Janeiro, RJ, Brasil. E-mail: biapasch@uerj.br

3 - CENPES/PETROBRAS, Rio de Janeiro, RJ. Brasil. E-mail: szatmari@cenpes.petrobras.com.br

4 - Grupo de Estudos em Petrologia (GEP/CNPq/UFRuralRJ), UFRuralRJ, Seropédica, RJ, Brasil. E-mail: sergio@ufrj.br
} 


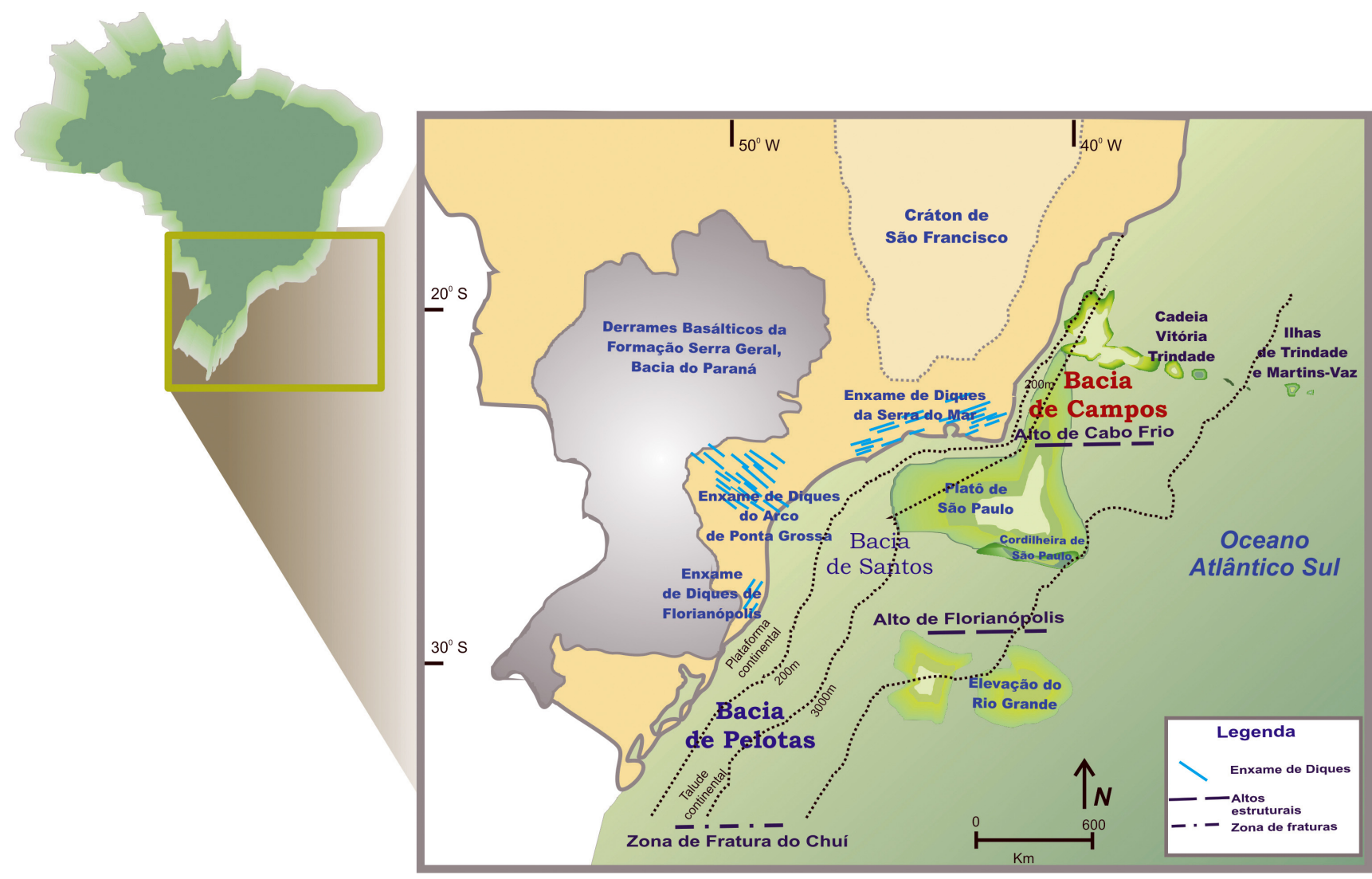

Figura 1 - Mapa de localização da bacia de Campos. No mapa destacam-se as principais feições fisiográficas da margem sudeste brasileira, além das principais ocorrências magmáticas do Cretáceo Inferior (e.g. Serra Geral, bacia do Paraná; Enxames de diques: Serra do Mar, Florianópolis e Arco de Ponta Grossa) (modificado de Lobo, 2000).

138 e $105 \mathrm{Ma}$, das bacias do Espírito Santo, Campos e Santos (Fodor \& Vetter 1984). Onde para estes autores, três grupos foram identificados com base na ampla variação de alguns elementos $\left(\mathrm{SiO}_{2}: 49-54 \%\right.$ de peso, $\mathrm{TiO}_{2}$ : 0,5 - 3,0\% de peso, $\mathrm{FeO}^{\mathrm{t}} / \mathrm{MgO}: 0,6-5,0 \mathrm{e}$ $\mathrm{La} / \mathrm{Yb}_{\mathrm{N}}: 1-6$ ) (Fodor \& Vetter 1984). Os grupos propostos foram: (i) basaltos enriquecidos em elementos incompatíveis, tais como $\mathrm{K}$ (algumas amostras com $\mathrm{K}_{2} \mathrm{O}>2,0 \%$ de peso), $\mathrm{Rb}$ (> $\left.18 \mathrm{ppm}\right), \mathrm{Zr}$ (> $\left.120 \mathrm{ppm}\right)$ e elementos terras raras leves. Algumas amostras deste grupo possuem $\mathrm{FeO}^{\mathrm{t}}=16 \%$ de peso e muitas delas possuem $\mathrm{SiO}_{2}=51-54 \%$ de peso; (ii) basaltos transicionais ou menos enriquecidos em elementos incompatíveis, tendo $\mathrm{K}_{2} \mathrm{O}<0,40 \%$ de peso e um monótono padrão de elementos terras raras; (iii) uma amostra de basalto empobrecido, com Ce $/ \mathrm{Yb}_{\mathrm{N}}=0,7$, onde $\mathrm{Ce}_{\mathrm{N}}=4$. Baseado nas descrições destes grupos, os basaltos enriquecidos foram classificados como P- e T-MORB (e.g. $\mathrm{Zr} / \mathrm{Nb} \cong 4-25$ ), os transicionais como T-MORB (e.g. $\mathrm{Zr} / \mathrm{Nb} \cong 8-27)$ e os empobrecidos como N-MORB (e.g. $\mathrm{Zr} / \mathrm{Nb}>30)$. Fodor \& Vetter (1984) concluíram que os basaltos da margem sudeste do Brasil referentes aquelas bacias estudadas foram extraídos de um manto heterogêneo com variáveis proporções de misturas de materiais de manto empobrecido (N-MORB) e pluma (P-MORB, e.g. o hot spot de Tristão da Cunha).

Baseado nas análises litogeoquímicas disponí- veis, Fodor (1987) identificou ambientes de geração de basaltos de alto- $\mathrm{TiO}_{2}$ e baixo- $\mathrm{TiO}_{2}$ para a margem sudeste do Brasil (bacias do Espírito Santo, Campos e Santos).

Os basaltos do Cretáceo Inferior foram estudados por Mizusaki et al. (1988) na bacia de Campos. As rochas vulcanoclásticas dos campos de Badejo, Linguado e Pampo foram classificadas como autoclásticas, piroclásticas e epiclásticas em função da gênese dos fragmentos. Através de um modelo vulcano-sedimentar foram delimitadas áreas de vulcanismo subaquoso e outras de vulcanismo subaéreo (acompanhado de manifestações explosivas). Este último é representado por camadas de tufos de cores avermelhadas que refletem períodos da exposição.

Análises de litogeoquímica e isotópicas ( $\mathrm{Sr}$ Nd) foram produzidas por Mizusaki et. al. (1992) para os basaltos do Cretáceo Inferior da bacia de Campos. Desta maneira, estes basaltos apresentaram valores de razões isotópicas similares aos basaltos do Paraná (Serra Geral), baixas concentrações de elementos incompatíveis e $\mathrm{TiO}_{2}<2 \%$ peso. Cálculos de fusão parcial em equilíbrio modal foi sugerido por Mizusaki et. al. (1992) onde uma quantidade de fusão parcial entre 9 e $25 \%$ de uma fonte mantélica do tipo granada peridotito foi requerida. Para estes autores a formação basáltica do Cretáceo Inferior da bacia de Campos teria sido extru- 
dida durante os primeiros estágios do grande processo do rifteamento do Gondwana ocorrido naquela região.

Recentemente, trabalhos abordando modelamento geoquímico quantitativo e caracterização de fonte mantélica estudos comparativos com a província Paraná-Etendeka foram desenvolvidos para as suítes basálticas das bacias de Campos e Pelotas (e.g. Lobo et al. 1999a, b; Lobo 2000; Lobo et al. 2001a,b; Lobo et al. 2006).

JUSTIFICATIVA O estudo da petrogênese dos basaltos do Cretáceo Inferior das bacias da margem sudeste do Brasil tem sido desenvolvida desde a década de oitenta com importantes trabalhos pioneiros (porém ainda com volume de dados ainda restrito) focando a geocronologia, litogeoquímica e geoquímica isotópica. $\mathrm{O}$ objetivo das pesquisas realizadas, em sua grande parte, tem sido de caracterização da fonte mantélica precursora (Fodor et al. 1983; Fodor \& Vetter 1984; Fodor 1987; Mizusaki et al. 1988; Mizusaki et. al. 1992; Lobo et al. 1999a, b; Lobo 2000; Lobo et al. 2001a,b; Lobo et al. 2006). Entretanto, o estudo de processos evolutivos ocorridos em câmara magmática foi pouco desenvolvido para qualquer uma das bacias em questão. No caso da bacia de Campos, o entendimento destes processos começou em Lobo (2000) com base em modelamento geoquímico quantitativo. Este modelo será apresentado a seguir. Esta proposta de estudo envolveu a compilação dos dados litogeoquímicos disponíveis na literatura (Fodor \& Vetter 1984; Mizusaki et. al. 1992) não sendo desenvolvido novos dados.

PETROGRAFIA Dezessete lâminas petrográficas da suíte basáltica de Campos foram selecionadas do arquivo CENPES/PETROBRAS e estudadas por Lobo (2000). A seguir apresenta-se um resumo dos resultados então obtidos.

O objetivo da descrição petrográfica foi o de caracterizar, de maneira geral, as principais fases mineralógicas, graus de cristalinidade, forma dos cristais, classificação granulométrica e textura. Especificamente, uma atenção maior foi dispensada para a assembléia de fenocristais os quais serviram como base para modelos geoquímicos descritos nos itens subseqüentes. Critérios disponíveis em Mackenzie et al. (1982) para a caracterização de basaltos toleíticos foram utilizados.

Os basaltos eocretáceos da bacia de Campos são, em geral, hipohialinos, inequigranulares porfiríticos, com fenocristais de granulação média $(\cong 2 \mathrm{~mm})$ envoltos por uma matriz granular fina $(<0,5 \mathrm{~mm})$ a vítrea (Fig. 2). A composição mineral da matriz é dada por plagioclásio, clinopiroxênio, olivina e minerais opacos. A assembléia de fenocristais é formada por plagioclásio, clinopiroxênio e olivina, tanto em grãos isolados quanto em aglomerados. Minerais secundários comuns são saussurita (principalmente encontrada nas bordas do plagioclásio), clorita e serpentina (alteração da olivina e cloritização da matriz). A matriz também é saussuritizada e devitrificada.

Aparentemente, há duas gerações de fenocris- tais de plagioclásio. Uma geração é representada por

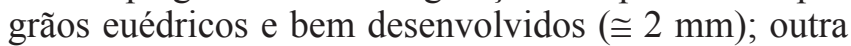
geração é representada por cristais finos ( $\cong 1 \mathrm{~mm})$ isolados na matriz, com geminação polissintética preservada, apesar de muito alterados e corroídos internamente. Os núcleos corroídos são manteados por uma segunda geração de plagioclásio livre de corrosão (Fig. 2a). Por vezes, observa-se também zonamento múltiplo. Microfenocristais de apatita são observados inclusos em grãos poiquilíticos tanto da primeira quanto da segunda geração de plagioclásio.

Os fenocristais de clinopiroxênio caracterizam-se por sua extinção oblíqüa e sua cor de interferência elevada. Ocorrem como cristais subédricos de granulação média. Feições de corrosão e manteamento também são observadas nestes fenocristais, muito embora com menos freqüência. Dois tipos de clinopiroxênio foram reconhecidos: augita e outro de cor natural castanha clara com boa clivagem em uma direção e fraturas perpendiculares, sendo, possivelmente, pigeonita (Fig. 2b). Os dois tipos de clinopiroxênio apresentamse, por vezes, em grãos poiquilíticos, englobando um mineral alterado, de relevo alto, cor verde e fraturado.

A olivina é o mineral menos freqüente nas assembléias de fenocristais estudadas. Apresenta-se muito alterada, subédrica e, às vezes, anédrica. Foi indentificada, fundamentalmente, por seu hábito preservado (Fig. 2c), já que, em geral, está totalmente substituída por serpentina e/ou clorita.

A matriz destes basaltos é geralmente fina (aproximadamente $0,5-0,8 \mathrm{~mm}$ ); por vezes vítrea ou devitrificada. É composta por plagioclásio, clinopiroxênio, olivina ( $<<1 \%$ de volume), minerais opacos subsólidus (subédrico e anédrico) e vidro vulcânico preenchendo interstícios entre os cristais (Fig. 2d).

A composição modal média da assembléia de fenocristais para estas rochas é, em \% de volume, a seguinte: $65 \%$ de plagioclásio, $30 \%$ clinopiroxênio e 5\% de olivina.

Algumas das características petrográficas descritas acima (e.g., a presença de dois tipos de clinopiroxênio, um rico em cálcio, augita, e outro pobre em cálcio, possivelmente pigeonita; percentagens de volume ínfimas de olivina na matriz, e caráter tardio dos minerais opacos) (Mackenzie et al., 1982) indicam o caráter toleítico da suíte basáltica da bacia de Campos. A tabela 1 apresenta um quadro sinóptico das características petrográficas dos basaltos do Eocretáceos da bacia de Campos.

\section{TRATAMENTO QUALITATIVO E APRESENTA- ÇÃO GERAL DOS DADOS GEOQUÍMICOS Va-} riações dos elementos maiores e dos elementos traços, incluindo os elementos terras raras, bem como variações isotópicas, foram estudadas, visando a análise dos possíveis processos petrogenéticos associados aos basaltos de Campos.

Dados geoquímicos foram préviamente selecionados da literatura (Fodor \& Vetter 1984 e Mizusaki et al. 1992) e estão apresentados na tabela 2. A compilação 

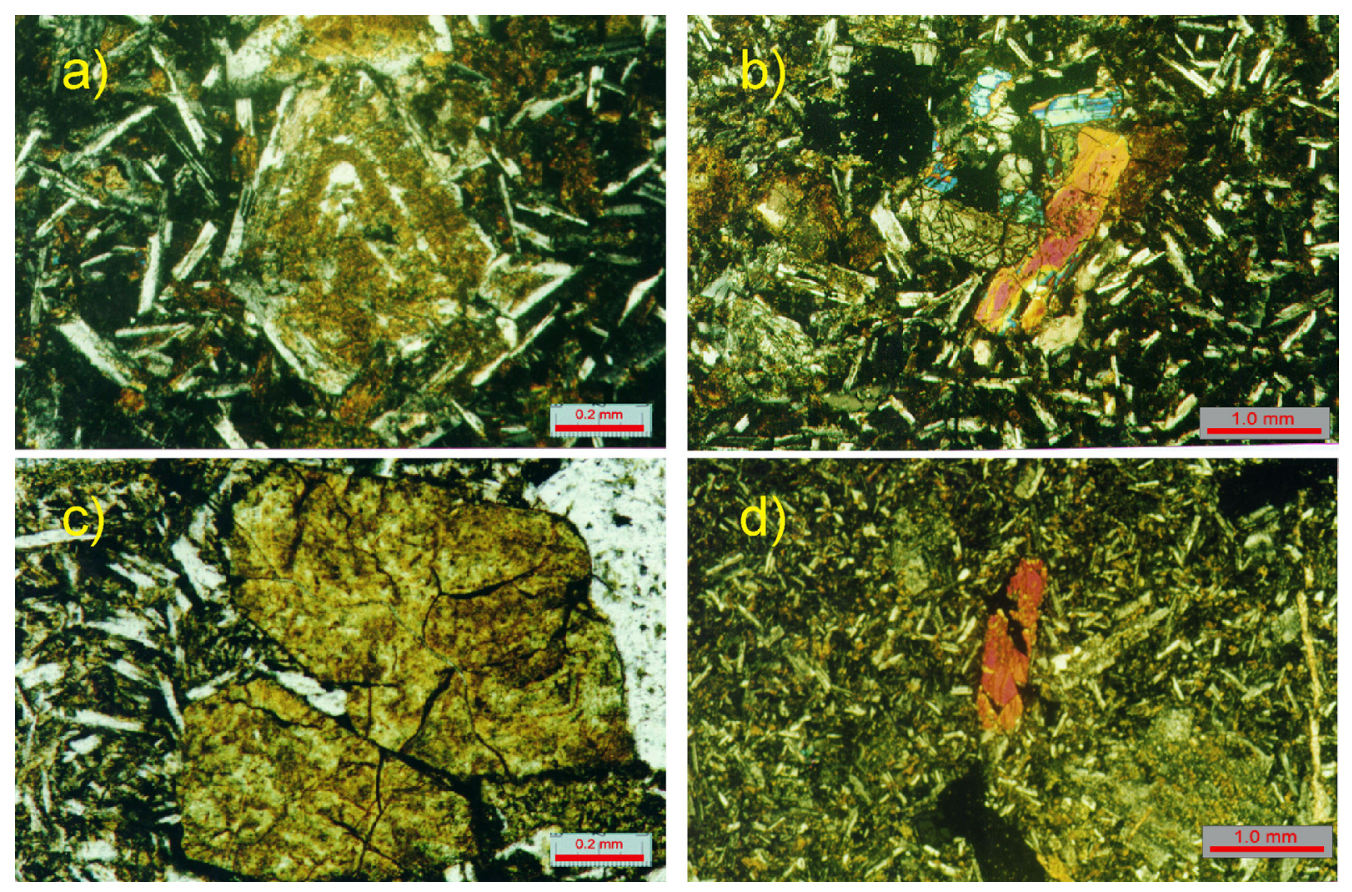

Figura 2 - Fotomicrografias dos basaltos do Cretáceo Inferior da bacia de Campos.

destes dados obedeceu aos seguintes critérios: (1) soma dos óxidos no intervalo 99 - 101\% de peso, (2) baixos valores de perda ao fogo e (3) norma CIPW para a caracterização dos toleítos (apresentação de hiperstênio normativo em todas as amostras).

Os dados compilados foram padronizados de forma a serem apresentados segundo seus valores originais e com o ferro expresso como ferro bivalente total (FeOt) (Tab. 2). Não foram produzidas novas análises geoquímicas como parte deste trabalho e trinta amostras fazem parte de toda a população dos basaltos de Campos.

CLASSIFICAÇÃO QUÍMICA E AMOSTRAS PARENTAIS As amostras são classificadas predominantemente como basalto, traquibasalto, traquiandesito basáltico e andesito basáltico no TAS (Fig. 3a) e no diagrama classificatório com base em elementos traços imóveis (Fig. 3b) abrangem os campos do basalto sub-alcalino, andesito basalto e andesito. As duas classificações são compatíveis com aquelas esperadas para rochas basálticas (Cox et al. 1979; Middlemost 1980). A classificação química com base em elementos altamente móveis como os álcalis $\left(\mathrm{Na}_{2} \mathrm{O}+\mathrm{K}_{2} \mathrm{O}\right.$; Fig. 3a) pode ser inapropriada para rochas ricas em potássio e, ainda, questionável para rochas vulcânicas alteradas devido à mobilização daqueles elementos em processos pós-magmáticos. No entanto, os resultados obtidos pela utilização dos diagramas com base em elementos traços incompatíveis (Fig. 3b) e o digrama com base no total de álcalis versus sílica (TAS; Fig. 3a) são similares e, portanto, sugerem que, se houve mobilização pós-magmática dos óxidos, este processo não afetou substancialmente a classificação química no TAS.

A afinidade toleítica dos basaltos do Cretáceo Inferior da bacia de Campos não é evidente no diagrama AFM (Fig. 3c), entretanto, o caráter toleítico é discriminado com base nos critérios petrográficos (dois tipos de clinopiroxênio e $<5 \%$ volume de olivina) e de classificação normativa (todas as amostras selecionadas possuem hiperstênio normativo) suportam esta classificação. Lobo (2000) demonstrou que a supressão de ferro das amostras da suíte basáltica do Cretáceo Inferior da bacia de Campos encontrada do diagrama AFM (Fig. $3 c)$ é devido sua anômala cristalização em ambiente de

Tabela 1 - Quadro sinóptico das características petrográficas dos basaltos do Cretáceo Inferior da bacia da Campos.

\begin{tabular}{l|c|c|c|c|c}
\hline $\begin{array}{l}\text { Textura } \\
\text { principal }\end{array}$ & $\begin{array}{c}\text { Granulação } \\
\text { da matriz }\end{array}$ & $\begin{array}{c}\text { Minerais da matriz } \\
\text { (em ordem de \%volume) }\end{array}$ & $\begin{array}{c}\text { Granulação } \\
\text { dos } \\
\text { fenocristais }\end{array}$ & $\begin{array}{c}\text { Fenocristais } \\
\text { (em ordem decrescente } \\
\text { de \%volume) }\end{array}$ & $\begin{array}{c}\text { Minerais } \\
\text { secundários }\end{array}$ \\
\hline $\begin{array}{l}\text { Porfirítica } \\
\begin{array}{l}\text { Matriz } \\
\text { intergranular e }\end{array}\end{array}$ & $\begin{array}{c}\text { fina }(0,5- \\
\text { intersertal }\end{array}$ & $\begin{array}{c}\text { plagioclásio, clinopiroxênio, } \\
\text { olivina, minerais opacos } \\
\text { subsólidus e vidro vulcânico }\end{array}$ & $\begin{array}{c}\text { média } \\
(\cong 2 \mathrm{~mm})\end{array}$ & $\begin{array}{c}\text { plagioclásio, } \\
\text { clinopiroxênio e } \\
\text { olivina }\end{array}$ & $\begin{array}{c}\text { saussurita, } \\
\text { clorita e } \\
\text { serpentina }\end{array}$ \\
\hline
\end{tabular}



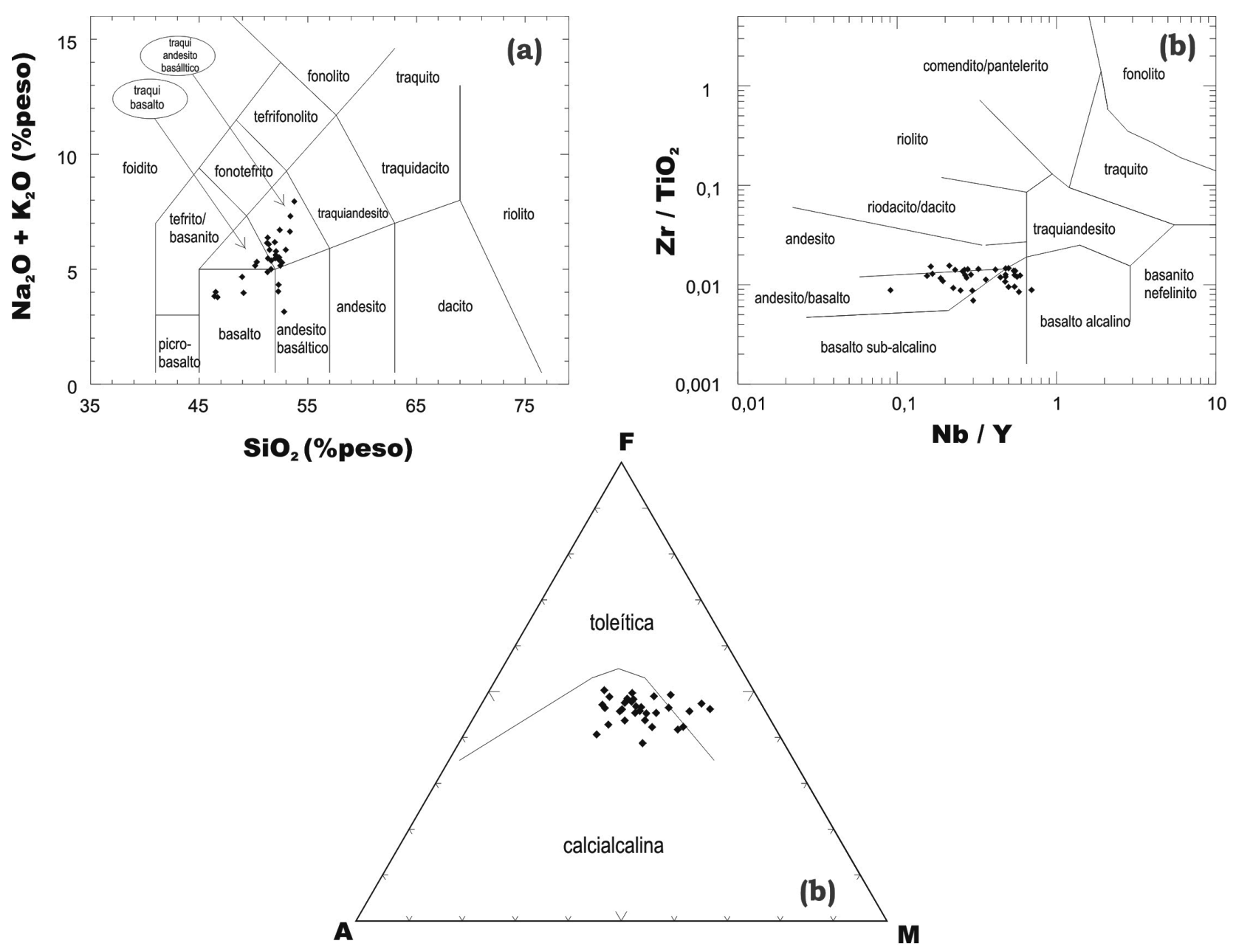

Figura 3 - Diagramas de classificação química de rochas: a) Le Maitre (1989); b) Winchester \& Floyd (1977) $e$ c) diagrama de classificação de series $\mathrm{AFM}\left(\mathrm{A}=\mathrm{Na}_{2} \mathrm{O}+\mathrm{K}_{2} \mathrm{O} ; \mathrm{F}=\mathrm{FeO}+0,9889 \mathrm{Fe}_{2} \mathrm{O}_{3} ; \mathrm{M}=\mathrm{MgO}\right)$.

alta fugacidade de oxigênio.

Amostras potencialmente parentais foram escolhidas obedecendo-se os seguintes critérios: 1) teores mais elevados de $\mathrm{MgO}$ acompanhados de valores de $\mathrm{Ni}$ e Cr elevados; e 2) teores relativamente baixos de $\mathrm{SiO}_{2}$. A tabela 3 apresenta seis amostras possívelmente representativas do magma parental. Destas, três possuem valores demasiadamente elevados de perda ao fogo (Li-4, RJS-139 e BD-15c; Tab. 3); duas possuem valores de $\mathrm{SiO}_{2}$ relativamente elevados (RJS-26 e BD-1a; Tab. 3) próximas de andesitos basaltos caracterizando-se como rochas muito evoluídas. Assim, a amostra RJS-156 foi considerada a melhor representante do magma parental da suíte basáltica de Campos. Ressalta-se que a amostra parental eleita (RJS-156) não representa um líquido primário, na medida em que apresenta baixos teores de $\mathrm{Ni}$ e Cr (Tab. 3).

\section{INVESTIGAÇ̃̃O DO PROCESSO EVOLUTI-}

VO O método dos mínimos quadrados foi aplicado na população das amostras dos basaltos de Campos o que possibilitou analisar estatisticamente qual a melhor curva de regressão se enquadraria para a população estudada nos diagramas bivariantes (elementos maiores e traços). O objetivo é de estudar possíveis processos pe- trogenéticos. $\mathrm{O} \mathrm{MgO}$ foi escolhido como o melhor índice de diferenciação devido a sua maior variabilidade (cerca de 3,80) em comparação com a da $\mathrm{SiO}_{2}$ (cerca de 1,24).

Diagramas de variação para elementos maiores e traços mostram valores de coeficientes de correlação polinomial $\left(\mathrm{R}^{2}\right)$ mais elevados que aqueles obtidos para correlações lineares (Fig.s 4 e 5; Tab. 4). Valores de R 2 para alguns óxidos e elementos traços são significantes acima de $99,9 \%$ para a quantidade de amostras plotada (Figs. 5c, d, e, f, h; Tab. 4), muito embora outros apresentem dispersão (Figs. 5b, i, j; Tab. 4). Este aspecto será considerado mais adiante. A tabela 4 apresenta a comparação entre os valores de $\mathrm{R}^{2}$ das funções lineares e polinomiais para os diagramas de variação dos elementos maiores e traços (os graus de significância das $\mathrm{R}^{2}$ polinomiais para $\mathrm{n}=30$ amostras e equações polinomiais de segunda ordem também são apresentadas). Os basaltos de Campos constituem uma única suíte do tipo baixo- $\mathrm{TiO}_{2}\left(\mathrm{TiO}_{2}=1,20 \pm 0,12 \%\right.$ de peso; Figs. 4 e $5 b)$.

A razão $\mathrm{La} / \mathrm{Yb}_{\mathrm{N}}$ da amostra parental RJS-156 é igual a 5,78 e a sua razão $\mathrm{La} / \mathrm{Nb}_{\mathrm{N}}$ é igual a 0,97 (Fig. 6). Manto litosférico subcontinental e a crosta continental possuem razões $\mathrm{La} / \mathrm{Nb}_{\mathrm{N}}>1$, enquanto que fontes astenosféricas possuem razões La/ $\mathrm{Nb}_{\mathrm{N}}<1$ (Thompson et 
Tabela 2 -Dados litogeoquímicos de Mizusaki et al. 1992 (referência 1) e Fodor e Vetter, 1984 (2).

\begin{tabular}{|c|c|c|c|c|c|c|c|c|c|c|c|c|c|c|c|c|c|c|}
\hline AMOSTRA & BD-8 & BD-4 & BD-11a & Li-4a & Li-4b & \begin{tabular}{|c|} 
RJS- \\
156 \\
\end{tabular} & $\begin{array}{c}\text { RJS- } \\
\text { 92a } \\
\end{array}$ & $\begin{array}{l}\text { RJS- } \\
\text { 92b }\end{array}$ & \begin{tabular}{|c|} 
RJS- \\
36a \\
\end{tabular} & \begin{tabular}{|c|} 
RJS- \\
36b \\
\end{tabular} & RJS-13 & BD-13 & \begin{tabular}{|c|} 
RJS- \\
315 \\
\end{tabular} & BD-3 & BD-1a & $\begin{array}{l}\text { BD- } \\
15 \mathrm{c}\end{array}$ & BD-2 & $\mathrm{Li}-4$ \\
\hline $\mathrm{SiO}_{2}$ & 52,19 & 51,36 & 50,91 & 43,55 & 42,70 & 45,07 & 50,80 & 52,74 & \begin{tabular}{|l|}
50,89 \\
\end{tabular} & 44,93 & 51,57 & \begin{tabular}{|l|}
48,39 \\
\end{tabular} & \begin{tabular}{|l|}
50,17 \\
\end{tabular} & 50,24 & 50,25 & 43,19 & 48,88 & 46,34 \\
\hline $\mathrm{TiO}_{2}$ & 1,24 & 1,02 & 1,23 & 1,30 & 1,23 & 1,49 & 1,34 & 1,23 & 1,05 & 1,44 & 1,27 & 1,28 & 1,12 & 1,25 & 1,33 & 1,19 & 1,23 & 1,27 \\
\hline $\mathrm{Al}_{2} \mathrm{O}_{3}$ & 15,35 & 15,05 & 15,36 & 13,95 & 12,59 & 16,23 & 14,12 & 15,37 & 14,73 & 14,28 & 14,11 & 14,05 & 15,90 & 14,44 & 14,86 & 13,45 & 14,23 & 14,46 \\
\hline $\mathrm{FeO}_{\mathrm{t}}$ & 9,95 & 9,31 & 9,91 & 11,00 & 10,69 & 11,31 & 10,03 & 9,77 & 10,65 & 12,82 & 10,13 & 9,25 & 9,46 & 9,78 & 9,44 & 11,51 & \begin{tabular}{|l|}
9,62 \\
\end{tabular} & 10,96 \\
\hline MnO & 0,22 & 0,13 & 0,18 & 0,33 & 0,18 & 0,24 & 0,14 & 0,20 & 0,21 & 0,24 & 0,12 & 0,23 & 0,20 & 0,21 & 0,10 & 0,18 & 0,27 & 0,27 \\
\hline MgO & 5,56 & 6,95 & 5,57 & 11,78 & 11,09 & 7,88 & 6,43 & 5,72 & 7,52 & 7,57 & 7,48 & 6,07 & 6,30 & 6,41 & 8,38 & 10,00 & 7,85 & 10,45 \\
\hline $\mathrm{CaO}$ & 9,28 & 9,43 & 9,52 & 6,11 & 6,64 & 9,53 & 9,62 & 8,05 & 6,32 & 10,76 & 7,50 & 8,68 & 8,61 & 8,28 & 4,29 & 9,19 & 7,21 & 5,85 \\
\hline $\mathrm{Na}_{2} \mathrm{O}$ & 3,14 & 2,16 & 2,91 & 2,03 & 2,32 & 2,92 & 2,04 & 2,19 & 4,79 & 3,52 & 3,01 & 3,19 & 2,88 & 2,69 & 2,49 & 2,13 & 2,46 & 2,34 \\
\hline $\mathrm{K}_{2} \mathrm{O}$ & 1,98 & 2,09 & 2,39 & 2,98 & 2,96 & 0,73 & 1,88 & 3,63 & 0,50 & 0,36 & 2,42 & 2,60 & 1,98 & 2,59 & 3,94 & 1,42 & 2,75 & 2,07 \\
\hline $\mathbf{P}_{2} \mathbf{O}_{5}$ & 0,18 & 0,13 & 0,17 & 0,15 & 0,11 & 0,38 & 0,17 & 0,16 & 0,15 & 0,13 & 0,18 & 0,19 & 0,19 & 0,17 & 0,17 & 0,12 & 0,16 & 0,15 \\
\hline PF & 1,73 & 2,46 & 1,98 & 6,98 & 9,34 & 4,86 & 2,12 & 0,94 & 3,60 & 4,24 & 2,70 & 5,47 & 2,62 & 3,77 & 4,73 & 7,80 & 4,69 & 5,70 \\
\hline total & \begin{tabular}{|l|}
100,81 \\
\end{tabular} & 100,08 & \begin{tabular}{|l|}
100,13 \\
\end{tabular} & 100,16 & \begin{tabular}{|l|l|}
99,84 \\
\end{tabular} & 100,66 & \begin{tabular}{|l|}
98,69 \\
\end{tabular} & \begin{tabular}{|l|}
99,99 \\
\end{tabular} & 100,42 & 100,29 & 100,48 & \begin{tabular}{|l|}
99,39 \\
\end{tabular} & \begin{tabular}{|l|}
99,43 \\
\end{tabular} & 99,83 & \begin{tabular}{|l|}
99,99 \\
\end{tabular} & 100,21 & 99,35 & 99,87 \\
\hline $\mathrm{Fe}_{2} \mathrm{O}_{3}$ & 4,07 & 6,09 & 4,92 & 5,39 & 5,53 & 8,17 & 6,77 & 5,58 & 4,94 & 6,19 & 7,72 & 5,60 & 4,43 & 6,23 & 6,64 & 7,35 & 6,46 & 5,48 \\
\hline $\mathrm{FeO}$ & 6,24 & 3,81 & 5,44 & 6,08 & 5,65 & 3,86 & 3,85 & 4,69 & 6,15 & 7,17 & 3,10 & 4,16 & 5,43 & 4,11 & 3,40 & 4,81 & 3,74 & 5,97 \\
\hline $\mathrm{Ni}$ & 63 & 72 & 60 & 40 & 44 & 113 & 56 & 64 & 56 & 74 & 59 & 52 & 62 & 58 & 80 & 61 & 58 & 46 \\
\hline $\mathrm{Cr}$ & 215 & 140 & 222 & 90 & 102 & 55 & 76 & 78 & 113 & 179 & 241 & 66 & 232 & 90 & 201 & 145 & 62 & 37 \\
\hline Ba & 505 & 504 & 656 & 985 & 1102 & 377 & 371 & 938 & 277 & 139 & 713 & 695 & 462 & 767 & 950 & 335 & 770 & 536 \\
\hline $\mathbf{R b}$ & 36 & 49 & 37 & 42 & 42 & 7 & 18 & 80 & 6 & 5 & 35 & 46 & 36 & 36 & 40 & 23 & 29 & 42 \\
\hline $\mathrm{Sr}$ & 272 & 312 & 305 & 248 & 241 & 411 & 304 & 280 & 411 & 301 & 261 & 365 & 301 & 348 & 297 & 309 & 355 & 306 \\
\hline \multicolumn{19}{|l|}{ Th } \\
\hline \multicolumn{19}{|l|}{ Sc } \\
\hline \multicolumn{19}{|l|}{ Co } \\
\hline \multicolumn{19}{|l|}{$\mathrm{Cu}$} \\
\hline \multicolumn{19}{|l|}{$\mathbf{Z n}$} \\
\hline $\mathrm{Nb}$ & 12 & 11 & 13 & 8 & 6 & 23 & 12 & 12 & 9 & 9 & 14 & 13 & 9 & 12 & 10 & 18 & 12 & 12 \\
\hline $\mathbf{Y}$ & 22 & 20 & 22 & 27 & 24 & 33 & 27 & 24 & 25 & 30 & 26 & 23 & 31 & 25 & 21 & 31 & 29 & 25 \\
\hline $\mathrm{Zr}$ & 155 & 140 & 152 & 114 & 108 & 132 & 159 & 180 & 118 & 100 & 176 & 154 & 142 & 182 & 143 & 101 & 175 & 161 \\
\hline La & 23,49 & \begin{tabular}{l|l|}
19,39 \\
\end{tabular} & 22,51 & 18,00 & 24,00 & 20,90 & 26,00 & 27,42 & 10,71 & 5,00 & 33,00 & 24,00 & 25,00 & 33,00 & 30,00 & 14,00 & 33,00 & 18,00 \\
\hline $\mathrm{Ce}$ & 49,59 & 44,86 & 52,56 & 40,00 & 50,00 & 46,54 & 55,00 & 59,00 & 27,79 & 12,01 & 63,00 & 49,00 & 56,00 & 67,00 & 57,00 & 27,00 & 58,00 & 44,00 \\
\hline \multicolumn{19}{|l|}{ Pr } \\
\hline Nd & 23,97 & 19,71 & 23,16 & 12,00 & 15,00 & 21,20 & 20,00 & 28,67 & 13,12 & 8,85 & 29,00 & 19,00 & 20,00 & 27,00 & 20,00 & 18,00 & 26,00 & 15,00 \\
\hline Sm & 5,42 & 4,38 & 5,12 & & & 4,83 & & 6,24 & 3,71 & 3,02 & & & & & & & & \\
\hline Eu & 1,55 & 1,30 & 1,45 & & & 1,54 & & 1,70 & 1,13 & 1,11 & & & & & & & & \\
\hline Gd & 4,93 & 3,84 & 4,82 & & & 4,53 & & 5,43 & 3,97 & 3,99 & & & & & & & & \\
\hline \multicolumn{19}{|l|}{ Tb } \\
\hline Dy & 4,47 & 3,68 & 4,26 & & & 4,56 & & 5,08 & 4,11 & 5,20 & & & & & & & & \\
\hline \multicolumn{19}{|l|}{ Ho } \\
\hline $\mathbf{E r}$ & 2,47 & 2,08 & 2,39 & & & 2,57 & & 2,79 & 2,32 & 3,40 & & & & & & & & \\
\hline $\mathbf{Y b}$ & 2,42 & 1,99 & 2,26 & & & 2,42 & & 2,64 & 2,30 & 3,27 & & & & & & & & \\
\hline Lu & 0,43 & 0,30 & 0,39 & & & 0,41 & & 0,49 & 0,37 & 0,53 & & & & & & & & \\
\hline${ }^{87} \mathrm{Sr} /{ }^{86} \mathrm{Sr}(\mathrm{m})$ & 0,70950 & 0,70945 & 0,70942 & 0,70957 & 0,70958 & 0,70586 & 0,70939 & 0,70936 & 0,70943 & 0,70847 & & & & & & & & \\
\hline $\begin{array}{c}{ }^{143} \mathbf{N d} /{ }^{144} \mathbf{N d} \\
(\mathrm{m})\end{array}$ & 0,51158 & 0,51145 & 0,51146 & 0,51154 & 0,51159 & 0,51217 & 0,51160 & 0,51154 & 0,51230 & 0,51263 & & & & & & & & \\
\hline $\begin{array}{l}\text { K-Ar rocha } \\
\text { total } 1\end{array}$ & & & & & & $\begin{array}{c}122+ \\
1-5 \\
\end{array}$ & & \begin{tabular}{|c}
$124+$ \\
$/-3$ \\
\end{tabular} & $\begin{array}{c}112+ \\
1-4 \\
\end{array}$ & & $\begin{array}{c}128+ \\
1-5 \\
\end{array}$ & & & $\begin{array}{c}124+ \\
1-7 \\
\end{array}$ & $\begin{array}{c}134+ \\
1-4 \\
\end{array}$ & $\begin{array}{c}111+ \\
/-13 \\
\end{array}$ & & \\
\hline $\begin{array}{l}\text { K-Ar rocha } \\
\text { Total } 2\end{array}$ & & & & & & & & & & & & & & $116+/-3$ & $126+/-3$ & & & \\
\hline Referência & 1 & 1 & 1 & 1 & 1 & 1 & 1 & 1 & 1 & 1 & 1 & 1 & 1 & 1 & 1 & 1 & 1 & 1 \\
\hline
\end{tabular}

\begin{tabular}{|c|c|c|c|c|c|c|c|c|c|c|c|c|c|c|c|c|c|c|}
\hline $\begin{array}{l}\text { AMOSTRA/ } \\
\text { CIPW }\end{array}$ & BD-8 & BD-4 & BD-11a & Li-4a & Li-4b & $\begin{array}{c}\text { RJS- } \\
156\end{array}$ & $\begin{array}{c}\text { RJS- } \\
\text { 92a }\end{array}$ & $\begin{array}{l}\text { RJS- } \\
\text { 92b }\end{array}$ & $\begin{array}{c}\text { RJS- } \\
36 \mathbf{a}\end{array}$ & $\begin{array}{c}\text { RJS- } \\
\mathbf{3 6 b}\end{array}$ & RJS-13 & BD-13 & \begin{tabular}{|c|} 
RJS- \\
315 \\
\end{tabular} & BD-3 & BD-1a & $\begin{array}{l}\text { BD- } \\
15 \mathrm{c}\end{array}$ & BD-2 & Li-4 \\
\hline Quartzo & 0,62 & 4,87 & 0 & 0 & 0 & 0 & 7,09 & 3,45 & 0 & 0 & 1,98 & 0 & 0 & 1,89 & 0 & 0 & 0,56 & 0 \\
\hline Hyperstenio & 11,3 & 10,41 & 38 & ( & 0 & 5,87 & 7,97 & 9,98 & 8,68 & 0 & 2,16 & & 14,19 & 9,51 & 0,54 & 2 & 14,72 & 15,58 \\
\hline Olivina & 0 & 0 & 0,32 & 23,5 & 19,71 & 5,96 & 0 & 0 & 8,16 & 9,88 & 0 & 5,2 & 0,16 & 0 & 0,16 & 12,2 & 0 & 10,6 \\
\hline Nefelina & 0 & 0 & 0 & 79 & 4 & 0 & 0 & 0 & 0 & 4,1 & & 0,48 & 0 & 0 & 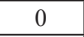 & 0 & 0 & 0 \\
\hline Ortoclásio & 11,79 & 12,59 & 14,37 & 18,84 & 19,24 & 4,46 & 11,46 & 21,55 & 3,06 & 2,17 & 14,51 & 16,31 & 12,08 & $\begin{array}{ll}15,88 \\
\end{array}$ & 4,33 & 9,05 & 17,05 & 12,95 \\
\hline Albita & 26,73 & 18,58 & 24,93 & 15,04 & 13,14 & 25,62 & 17,75 & 18,6 & 41,7 & 23,22 & 25,88 & 27,72 & 25,02 & 23,56 & \begin{tabular}{ll|}
21,94 \\
\end{tabular} & \begin{tabular}{|l|}
19,41 \\
\end{tabular} & 21,86 & 20,96 \\
\hline Anortita & 22,07 & 25,71 & 22,14 & 21,56 & 16,76 & 30,1 & 24,54 & 21,54 & 17,69 & 22,94 & 18,15 & 17,31 & 25,34 & 20,38 & 18,58 & 24,66 & 20,69 & 24,12 \\
\hline \multicolumn{19}{|l|}{ Leucita } \\
\hline Diopsidio & 18,85 & 16,68 & 19,97 & 8,06 & 15,1 & 12,9 & 18,4 & 14,18 & 11,01 & 25,28 & 14,61 & 21,48 & 14,02 & 16,68 & 2,2 & 18,58 & 12,57 & 4,56 \\
\hline \multicolumn{19}{|l|}{ Acmita } \\
\hline Magnetita & 5,93 & 8,99 & 7,23 & 8,34 & 8,81 & 9,25 & 9,23 & 8,13 & 7,36 & 9,29 & 6,76 & 8,6 & 6,61 & 9,35 & 7,74 & 11,45 & 9,83 & 8,39 \\
\hline Hematita & 0 & 0 & 0 & 0 & 0 & 2, & 0 , & 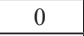 & 0 & 0 & 3,18 & 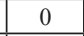 & 0 & 0 & 1,59 & 0 & 0 & 0 \\
\hline Ilmenita & 2,36 & 1,96 & 2,36 & 2,65 & 2,5 & 2,94 & 2,62 & 2,34 & 2,04 & 2,83 & 2,45 & 2,57 & 2,19 & 2,45 & 2,64 & 2,43 & 2,45 & 2,55 \\
\hline Apatita & 0,43 & 0,31 & 0,4 & 0,38 & 0,29 & 0,94 & 0,43 & 0,38 & 0,38 & 0,33 & 0,43 & 0,47 & 0,47 & 0,43 & 0,43 & 0,31 & 0,4 & 0,38 \\
\hline \multicolumn{19}{|l|}{ Wollastonita } \\
\hline Total & 100,08 & 100,1 & 100,1 & 100,16 & 100,17 & 100,13 & 100,09 & 100,15 & 100,08 & 100,04 & 100,11 & 100,14 & 100,08 & 100,13 & 100,15 & 100,09 & 100,13 & 100,09 \\
\hline
\end{tabular}


Tabela 2 (cont.)

\begin{tabular}{|c|c|c|c|c|c|c|c|c|c|c|c|c|c|c|c|c|c|c|}
\hline AMOSTRA & Li-7 & $\begin{array}{c}\text { RJS- } \\
139\end{array}$ & PM-3 & \begin{tabular}{|c|} 
RJS- \\
159 \\
\end{tabular} & \begin{tabular}{|l|} 
CST- \\
1-RJ \\
\end{tabular} & \begin{tabular}{|l|} 
RJS-92 \\
3_1/12 \\
\end{tabular} & \begin{tabular}{|c|} 
RJS-92 \\
3_9/12
\end{tabular} & \begin{tabular}{|l} 
RJS-92 \\
$4 \_3 / 11$ \\
\end{tabular} & \begin{tabular}{|c|} 
RJS-92 \\
$5 \_1 / 11$
\end{tabular} & \begin{tabular}{|c|} 
RJS-92 \\
5 5_/11
\end{tabular} & $\begin{array}{c}\text { RJS-92 } \\
6 \_3 / 13\end{array}$ & \begin{tabular}{|l|} 
RJS-92 \\
6_10/13 \\
\end{tabular} & \begin{tabular}{|c|} 
BD-1a \\
$5 \_1 / 2$ \\
\end{tabular} & \begin{tabular}{|l|} 
BD-2 \\
5_1/7 \\
\end{tabular} & \begin{tabular}{|l|} 
BD-2 \\
3_7/7 \\
\end{tabular} & \begin{tabular}{|c|} 
BD-2 \\
$6 \_11 / 13$ \\
\end{tabular} & \begin{tabular}{|c|} 
RJS-13 \\
$9 \_3 / 3$ \\
\end{tabular} & $\begin{array}{c}\text { RJS-26 } \\
4 \_1 / 1\end{array}$ \\
\hline $\mathrm{SiO}_{2}$ & 50,44 & 45,41 & 46,41 & 44,35 & 33,35 & 50,61 & 50,01 & 49,30 & 50,59 & 51,97 & 50,08 & 51,16 & 50,24 & 52,22 & 51,35 & 52,41 & 51,88 & 51,43 \\
\hline $\mathrm{TiO}_{2}$ & 1,33 & 1,34 & 1,21 & 1,14 & 0,82 & 1,09 & 1,10 & 1,15 & 1,17 & 1,12 & 1,26 & 1,10 & 1,24 & 1,01 & 1,13 & 1,13 & 1,07 & 1,03 \\
\hline $\mathrm{Al}_{2} \mathrm{O}_{3}$ & 13,91 & 11,73 & 13,63 & 11,07 & 14,65 & 15,23 & 15,03 & 16,06 & 16,45 & 16,26 & 15,14 & 15,07 & 15,34 & 15,34 & 15,01 & 14,67 & 15,08 & 10,88 \\
\hline $\mathrm{FeO}_{\mathrm{t}}$ & 10,36 & 10,61 & 9,88 & 9,81 & 7,87 & 9,75 & 10,20 & 10,05 & 10,07 & 9,47 & 10,27 & 9,50 & 10,66 & 8,84 & 11,00 & 9,46 & 10,48 & 12,45 \\
\hline MnO & 0,14 & 0,10 & 0,14 & 0,14 & 0,12 & 0,14 & 0,09 & 0,24 & 0,22 & 0,14 & 0,20 & 0,15 & 0,12 & 0,17 & 0,20 & 0,11 & 0,22 & 0,10 \\
\hline MgO & 6,73 & 10,04 & 7,54 & 3,15 & 9,82 & 4,83 & 6,27 & 6,06 & 5,66 & 4,71 & 4,57 & 5,04 & 6,49 & 5,63 & 7,02 & 6,05 & 5,87 & 11,38 \\
\hline $\mathrm{CaO}$ & 7,12 & 5,82 & \begin{tabular}{|l|}
11,32 \\
\end{tabular} & 13,83 & 6,62 & 9,50 & 7,17 & 8,87 & 8,55 & 9,26 & 8,82 & 6,27 & 5,71 & 5,12 & 6,09 & 5,82 & 8,26 & 5,05 \\
\hline $\mathrm{Na}_{2} \mathrm{O}$ & 2,82 & 1,98 & 2,08 & 1,84 & 1,15 & 2,56 & 2,78 & 2,97 & 2,30 & 2,69 & 2,85 & 2,93 & 2,13 & 2,37 & 2,38 & 3,73 & 2,80 & 1,91 \\
\hline $\mathrm{K}_{2} \mathrm{O}$ & 2,25 & 2,69 & 1,67 & 4,07 & 1,14 & 3,19 & 3,42 & 2,25 & 2,51 & 3,49 & 3,07 & 3,43 & 3,29 & 5,35 & 3,31 & 3,44 & 2,57 & 1,16 \\
\hline $\mathbf{P}_{2} \mathbf{O}_{5}$ & 0,19 & 0,18 & 0,17 & 0,17 & 0,11 & 0,17 & 0,15 & 0,16 & 0,18 & 0,18 & 0,17 & 0,16 & 0,17 & 0,14 & 0,17 & 0,17 & 0,20 & 0,17 \\
\hline PF & 5,18 & 8,95 & 6,05 & 10,82 & 23,80 & 3,59 & 4,68 & 2,71 & 2,76 & 2,59 & 3,32 & 3,89 & 3,89 & 3,33 & 2,33 & 4,49 & 1,48 & 4,37 \\
\hline total & 100,47 & \begin{tabular}{|l|l|}
98,84 \\
\end{tabular} & 100,11 & 100,38 & 99,45 & 100,66 & 100,91 & 99,82 & 100,45 & \begin{tabular}{|l|l|}
101,87 \\
\end{tabular} & 99,75 & 98,71 & \begin{tabular}{|l|}
99,27 \\
\end{tabular} & \begin{tabular}{|l|}
99,53 \\
\end{tabular} & 100,00 & \begin{tabular}{|l|}
101,49 \\
\end{tabular} & 99,91 & 99,93 \\
\hline $\mathrm{Fe}_{2} \mathrm{O}_{3}$ & 6,08 & 7,24 & 5,52 & 7,51 & 6,81 & 8,92 & 7,25 & 6,25 & 6,78 & 5,27 & 6,36 & 6,63 & 6,94 & 6,50 & 7,43 & 7,18 & 6,40 & 12,51 \\
\hline $\mathrm{FeO}$ & 4,82 & 4,01 & 4,85 & 2,97 & 4,27 & 2,08 & 4,17 & 4,70 & 4,24 & 4,97 & 4,89 & 3,92 & \begin{tabular}{|l|}
4,89 \\
\end{tabular} & 3,30 & 4,50 & 3,44 & 4,82 & 1,75 \\
\hline $\mathbf{N i}$ & 46 & 51 & 76 & 39 & 76 & 60 & 43 & 43 & 60 & 55 & 47 & 66 & 55 & 44 & 53 & 56 & 57 & 99 \\
\hline $\mathrm{Cr}$ & 37 & 138 & 235 & 45 & 174 & 250 & 85 & 70 & 60 & 50 & 35 & 80 & 55 & 45 & 65 & 75 & 255 & 410 \\
\hline Ba & 536 & 727 & 463 & 634 & 603 & 440 & 710 & 365 & 585 & 565 & 575 & 420 & 620 & 965 & 750 & 660 & 705 & 275 \\
\hline $\mathbf{R b}$ & 42 & 19 & 35 & 74 & 23 & 30 & 65 & 31 & 44 & 52 & 41 & 37 & 48 & 50 & 46 & 36 & 37 & 30 \\
\hline $\mathrm{Sr}$ & 306 & 287 & 363 & 252 & 215 & 325 & 255 & 270 & 360 & 300 & 260 & 260 & 330 & 320 & 280 & 290 & 285 & 95 \\
\hline Th & & & & & & & & 5 & 5 & & 5 & & & & 5 & & & \\
\hline Sc & & & & & & & 38 & 32 & & 37 & & & & 32 & & 41 & & \\
\hline Co & & & & & 46 & 49 & 60 & 69 & 63 & 67 & 62 & 54 & 59 & 56 & 90 & 57 & 60 & \\
\hline $\mathrm{Cu}$ & & & & & 108 & 207 & 122 & 125 & 210 & 140 & 140 & 129 & 93 & 143 & 132 & 87 & 89 & \\
\hline $\mathbf{Z n}$ & & & & & 75 & 127 & 78 & 102 & 125 & 125 & 75 & 111 & 67 & 149 & 85 & 89 & 57 & \\
\hline $\mathrm{Nb}$ & 12 & 12 & 8 & 9 & 2 & 6 & 6 & 9 & 10 & 9 & 6 & 6 & 11 & 7 & 9 & 7 & 9 & 9 \\
\hline $\mathbf{Y}$ & 25 & 22 & 30 & 18 & 22 & 32 & 39 & 33 & 36 & 39 & 36 & 31 & 34 & 33 & 35 & 43 & 34 & 40 \\
\hline $\mathrm{Zr}$ & 161 & 128 & 152 & 108 & 72 & 127 & 135 & 135 & 167 & 158 & 162 & 119 & 178 & 156 & 155 & 172 & 152 & 96 \\
\hline $\mathrm{La}$ & 18,00 & 16,00 & 24,00 & 4,00 & 17,00 & & & 21,30 & 26,50 & & 29,90 & & & & 26,80 & & & \\
\hline $\mathrm{Ce}$ & \begin{tabular}{|l|l|}
44,00 \\
\end{tabular} & 43,00 & \begin{tabular}{|l|l|}
44,00 \\
\end{tabular} & 9,00 & 32,00 & & & 51,00 & \begin{tabular}{|l|}
51,30 \\
\end{tabular} & & 61,30 & & & & \begin{tabular}{|l|l|}
53,70 \\
\end{tabular} & & & \\
\hline \multicolumn{19}{|l|}{$\mathrm{Pr}$} \\
\hline Nd & 15,00 & 20,00 & 23,00 & 4,00 & 18,00 & & & 36,30 & 42,90 & & 40,90 & & & & \begin{tabular}{|l|} 
\\
\end{tabular} & & & \\
\hline Sm & & & & & & & & 4,40 & 5,60 & & 5,40 & & & & \begin{tabular}{|l|}
5,10 \\
\end{tabular} & & & \\
\hline Eu & & & & & & & & 1,40 & 1,40 & & 1,40 & & & & 1,50 & & & \\
\hline \multicolumn{19}{|l|}{ Gd } \\
\hline Tb & & & & & & & & 0,98 & 1,40 & & 1,10 & & & & \begin{tabular}{l|l}
0,82 \\
\end{tabular} & & & \\
\hline \multicolumn{19}{|l|}{ Dy } \\
\hline \multicolumn{19}{|l|}{ Ho } \\
\hline \multicolumn{19}{|l|}{$\mathrm{Er}$} \\
\hline $\mathbf{Y b}$ & & & & & & & & 2,50 & 2,70 & & 3,40 & & & & 2,80 & & & \\
\hline Lu & & & & & & & & 0,41 & 0,48 & & 0,47 & & & & 0,42 & & & \\
\hline \multicolumn{19}{|l|}{${ }^{87} \mathrm{Sr} /{ }^{86} \mathrm{Sr}(\mathrm{m})$} \\
\hline \multicolumn{19}{|c|}{${ }^{143} \mathrm{Nd} / /^{144} \mathrm{Nd}(\mathrm{m})$} \\
\hline $\begin{array}{l}\text { K-Ar rocha } \\
\text { total } 1\end{array}$ & & $122+/-4$ & & & & & & & & & & & & & & & & \\
\hline \multicolumn{19}{|l|}{$\begin{array}{c}\text { K-Ar rocha } \\
\text { total } 2\end{array}$} \\
\hline Referência & 1 & 1 & 1 & 1 & 1 & 2 & 2 & 2 & 2 & 2 & 2 & 2 & 2 & 2 & 2 & 2 & 2 & 2 \\
\hline
\end{tabular}

\begin{tabular}{|c|c|c|c|c|c|c|c|c|c|c|c|c|c|c|c|c|c|c|}
\hline AMOSTR & Li-7 & $\begin{array}{c}\text { RJS- } \\
139 \\
\end{array}$ & PM-3 & \begin{tabular}{c|} 
RJS- \\
159 \\
\end{tabular} & \begin{tabular}{|l|} 
CST- \\
1-RJ \\
\end{tabular} & \begin{tabular}{|c|} 
RJS-92 \\
3_1/12 \\
\end{tabular} & \begin{tabular}{|l|} 
RJS-92 \\
3_9/12 \\
\end{tabular} & \begin{tabular}{|c|} 
RJS-92 \\
$4 \_3 / 11$ \\
\end{tabular} & \begin{tabular}{|c|} 
RJS-92 \\
$5 \_1 / 11$ \\
\end{tabular} & \begin{tabular}{|c|} 
RJS-92 \\
$5 \_7 / 11$ \\
\end{tabular} & \begin{tabular}{|c|} 
RJS-92 \\
$6 \_3 / 13$ \\
\end{tabular} & \begin{tabular}{|l|} 
RJS-92 \\
6_10/13 \\
\end{tabular} & \begin{tabular}{|l|} 
BD-1a \\
$5 \_1 / 2$ \\
\end{tabular} & \begin{tabular}{|l|} 
BD-2 \\
$5 \_1 / 7$ \\
\end{tabular} & \begin{tabular}{|l|} 
BD-2 \\
3_7/7 \\
\end{tabular} & \begin{tabular}{|c|} 
BD-2 \\
$6 \_11 / 13$ \\
\end{tabular} & \begin{tabular}{|c|} 
RJS-13 \\
$9 \_3 / 3$ \\
\end{tabular} & \begin{tabular}{|c|} 
RJS-26 \\
$4 \_1 / 1$ \\
\end{tabular} \\
\hline Quart & 3,21 & 0 & 0 & 0 & 0 & 1,91 & 0 & 0 & 4,11 & 0,02 & 0,53 & 3,11 & 4,26 & 0,86 & 3,32 & 0 & 2,87 & 10,16 \\
\hline Hypersten & 13,43 &, 07 & 4,7 & 0 & \begin{tabular}{|l|}
3,66 \\
\end{tabular} & 86 & 9,39 & 63 & 9,71 & 6,16 & 4,81 & 8,65 & \begin{tabular}{|l|}
16,37 \\
\end{tabular} & 10,94 & \begin{tabular}{|l|}
15,09 \\
\end{tabular} & 8,69 & 9,85 & 26,76 \\
\hline Olivina & 0 & 1,74 & 3,68 & 0 & 19,94 & 0 & 0,42 & 2,51 & 0 & 0 & 0 & & 0 & 0 & 0 & 0,84 & 0 & 0 \\
\hline Nefeli & 0 & 0 & 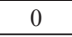 & 4,44 & 0 & 0 & 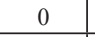 & 0 & 0 & 0 & 0 & 0 & 0 & 0 & 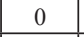 & 0 & 0 & 0 \\
\hline$\overline{\text { Ortoclá }}$ & 13,89 & \begin{tabular}{l|l|}
7,56 \\
\end{tabular} & 10,48 & 26,66 & 8,57 & 19,2 & 0,79 & 13,56 & 15,05 & 20,63 & 18,67 & 1,15 & 20,13 & 32,6 & \begin{tabular}{|l|}
19,86 \\
\end{tabular} & 20,73 & 15,34 & 7,03 \\
\hline Albita & 91 & 49 & 18 & 3 & 12,34 & 21,99 & 4,18 & 61 & 68 & 75 & 4,77 & 5,86 & \begin{tabular}{|l|}
18,69 \\
\end{tabular} & 20,62 & \begin{tabular}{|l|}
20,45 \\
\end{tabular} & 32,13 & 3,87 & 16,62 \\
\hline Anortita &, 49 & 8,82 & 4,25 & 03 & 39,94 & 21,03 & 9 & ,38 & 27,57 & ,99 & 19,97 & 8,64 & \begin{tabular}{|l|}
23,44 \\
\end{tabular} & 15,91 & \begin{tabular}{|l|}
20,82 \\
\end{tabular} & 13,41 & 21 & 18,18 \\
\hline \multicolumn{19}{|l|}{ Leucita } \\
\hline Diopsidio & 12,86 & 97 & 27,06 & 18,79 & 0,84 & 20,24 & 15 & 15,54 & 11,35 & 39 & 19,04 &, 09 & 3,94 & 7,59 & 7,08 & 1,8 & 15,09 & 5,06 \\
\hline \multicolumn{19}{|l|}{ Acmita } \\
\hline Magnetita & 9,2 & \begin{tabular}{ll|}
10,33 \\
\end{tabular} & 8,46 & 7,48 & 12,52 & 4,07 & 10,79 & 9,24 & 9,96 & 7,64 & 9,47 & 10,03 & \begin{tabular}{|l|}
10,42 \\
\end{tabular} & \begin{tabular}{|l|}
8,56 \\
\end{tabular} & \begin{tabular}{|l|}
10,92 \\
\end{tabular} & 8,39 & 9,36 & 3,01 \\
\hline Hematita & 0 & 0,87 & 0 & \begin{tabular}{|l|}
3,16 \\
\end{tabular} & 0 & 6,26 & 0 & 0 & 0 & 0 & 0 & 0 & 0 & \begin{tabular}{|l|}
0,78 \\
\end{tabular} & 0 & 1,53 & 0 & 10,77 \\
\hline Ilmenita & 2,64 & 2,81 & 2,44 & 2,39 & 1,97 & 2,1 & 2,14 & 2,22 & 2,25 & 2,13 & 2,45 & 2,17 & 2,44 & 1,96 & 2,18 & 2,18 & 2,06 & 2,01 \\
\hline Apatita & 0,47 & 0,47 & 0,42 & 0,45 & 0,34 & 42 & 37 & 0 &, 45 & 0,42 &, 42 & 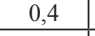 & 0,43 & 0,33 & 0,4 & 42 & 0,47 & 0,42 \\
\hline Wollastonita & & & & 16,69 & & & & & & & & & & & & & & \\
\hline Total & 100,1 & 100,13 & 100,09 & 100,12 & 100,12 & 100,08 & 100,13 & 100,07 & \begin{tabular}{|l|}
100,13 \\
\end{tabular} & 100,13 & \begin{tabular}{|l|}
100,13 \\
\end{tabular} & 100,1 & 100,12 & 100,15 & 100,12 & \begin{tabular}{|l|}
100,12 \\
\end{tabular} & \begin{tabular}{|l|}
100,12 \\
\end{tabular} & 100,02 \\
\hline
\end{tabular}


Tabela 3 - Tabela comparativa entre as seis amostras que apresentam os maiores valores de MgO.

\begin{tabular}{l|c|c|c|c|c}
\hline Amostra & MGO (\% de peso) & SIO $_{2}$ (\% de peso) & NI (ppm) & CR (ppm) & PF (\% de peso) \\
\hline RJS-26 & 11,38 & 51,43 & 99 & 410 & 4,37 \\
\hline LI -4 & 10,45 & 46,34 & 46 & 37 & 5,70 \\
\hline RJS-139 & 10,04 & 45,41 & 51 & 138 & 8,95 \\
\hline BD-15C & 10,00 & 43,19 & 61 & 145 & 7,80 \\
\hline BD-1 & 8,38 & 50,25 & 80 & 201 & 4,73 \\
\hline RJS-156 & 7,88 & 45,07 & 113 & 55 & 4,86 \\
\hline
\end{tabular}

al. 1982). A razão inferior que a unidade encontrada para a parental da suíte basáltica de Campos é indicativo de amostra sem contribuição litosférica substancial. Desta maneira, a RJS-156 representa, não somente o líquido parental, como, também, o líquido parental não contaminado (Fig. 6).

É valido comentar que, embora alguns diagramas de variação demonstraram dispersão, a média da razão $\mathrm{La} / \mathrm{Yb}_{\mathrm{N}}$ de toda a população analisada é de 5,88 e o desvio padrão é de 1,09 considerando-se uma população com trinta indivíduos. Isto suporta o fato de que todas as amostras são pertencentes a um único conjunto cogenético e que seu processo evolutivo é investigado naqueles digramas por somente uma curva.

Cristalização fracionada pura e simples (com mudança da assembléia fracionante) e assimilação concomitante a cristalização fracionada $(A F C)$ resultam em funções polinomiais (curvilineares) e são expressas em diagramas bivariante por curvas com inflexões. Os dois processos comentados são indistintos em diagramas bivariantes. Assim, os possíveis processos evolutivos para a suíte basáltica de Campos, com os dados apresentados até o momento, seriam os de cristalização fracionada pura e simples e o de $A F C$.

Cristalização fracionada pura e simples não produz variações nas razões de elementos traços incompatíveis entre a amostra parental e derivativa de uma determinada suíte maiores que uma vez e meia (e.g. Allégre \& Minster 1978). Razões entre $\mathrm{Zr}, \mathrm{Nb}$ e Y foram utilizadas para chegar a variação entre a amostra mais

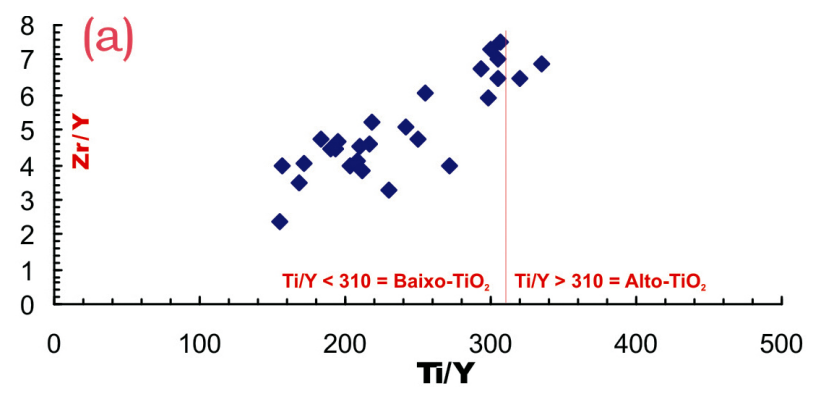

Figura 4 - Diagramas bivariantes entre razões de elementos traços incompativeis $(\mathrm{Zr} / \mathrm{Y}$ versus $\mathrm{Ti} / \mathrm{Y})$ mostrando a relação limitrofe entre alto- $\mathrm{TiO} 2(\mathrm{Ti} / \mathrm{Y}<310)$ e baixo-TiO2 (Ti/Y>310) (Hergt et al, 1991; Peate, et al. 1992) evoluída (RJS-92_3/13) e a amostra parental (RJS-156) da formação basáltica do Cretáceo Inferior da bacia de Campos (Tab. 5). As razões $\mathrm{Zr} / \mathrm{Nb}$ aumentam cerca de cinco vezes e meia e a razão $\mathrm{Y} / \mathrm{Nb}$ varia cerca de quatro vezes. Tais variação não conferem ás condições esperadas ao processo de cristalização fracionada pura e simples.

\section{MODELAMENTO GEOQUÍMICO QUANTITA- TIVO DO PROCESSO EVOLUTIVO GERADOR} DOS BASALTOS DE CAMPOS Os valores mais elevados de $\mathrm{R}^{2}$ das funções polinomiais (Figs. 4 e 5; Tab. 4) indicam cristalização fracionada como um provável processo petrogenético evolutivo para os basaltos do Cretáceo Inferior da bacia de Campos. Entretanto, as amplas variações de razões de alguns elementos traços incompatíveis citadas anteriormente implicam que a petrogênese dos basaltos de Campos deve ter envolvido processos que não seriam exclusivamente cristalização fracionada. Não há evidências petrográficas ou de campo que suportem processos de mistura magmática para os basaltos de Campos. Os processos evolutivos de mistura magmática e assimilação simples são representados, em diagramas bivariantes, como funções lineares com a presença de hiatos composicionais. Tais relações foram excluídas na suíte basáltica de Campos através da análise estatística realizada com o método dos mínimos quadrados (Figs. 4 e 5; Tab. 4).

Descartados os processos de mistura magmática e assimilação simples, bem como o de cristalização fracionada exclusiva, assimilação concomitante à cristalização fracionada $(A F C)$ passa a ser o processo petrogenético evolutivo mais provável para os basaltos de Campos. Isto é suportado pelas amplas variações entre elementos traços incompatíveis e imóveis (Tab. 5).

$\mathrm{O}$ modelamento quantitativo do processo evolutivo de $A F C$ proposto por DePaolo (1981) descreve a evolução composicional de um magma através das seguintes equações:

$$
\begin{aligned}
& \mathrm{C}_{\mathrm{L}} / \mathrm{C}_{\mathrm{O}}=(\mathrm{r} /(\mathrm{r}-1+\mathrm{D})) \cdot\left(\mathrm{C}_{\mathrm{a}} / \mathrm{C}_{\mathrm{O}} \cdot(1-\mathrm{f})\right)+\mathrm{f} \quad(\text { equação } 3 \mathrm{a}) ; \\
& \left.\varepsilon_{\mathrm{L}}=\left(\varepsilon_{\mathrm{O}}\right)+\left(\left(\varepsilon_{\mathrm{a}}-\varepsilon_{\mathrm{O}}\right)\right) \cdot\left(1-\left(\left(\mathrm{C}_{\mathrm{O}} / \mathrm{C}_{\mathrm{L}}\right) \cdot(\mathrm{f})\right)\right) \text { (equação } 3 \mathrm{~b}\right), \\
& \text { onde } \mathrm{f}=\mathrm{F}^{-(\mathrm{r}-1+\mathrm{D}) /(\mathrm{r}-1)} ; \mathrm{C}_{\mathrm{L}} \text { e } \varepsilon_{\mathrm{L}} \text { são, respectivamen- }
\end{aligned}
$$
te, as concentrações de elementos traços e composição isotópica de um magma contaminado. 

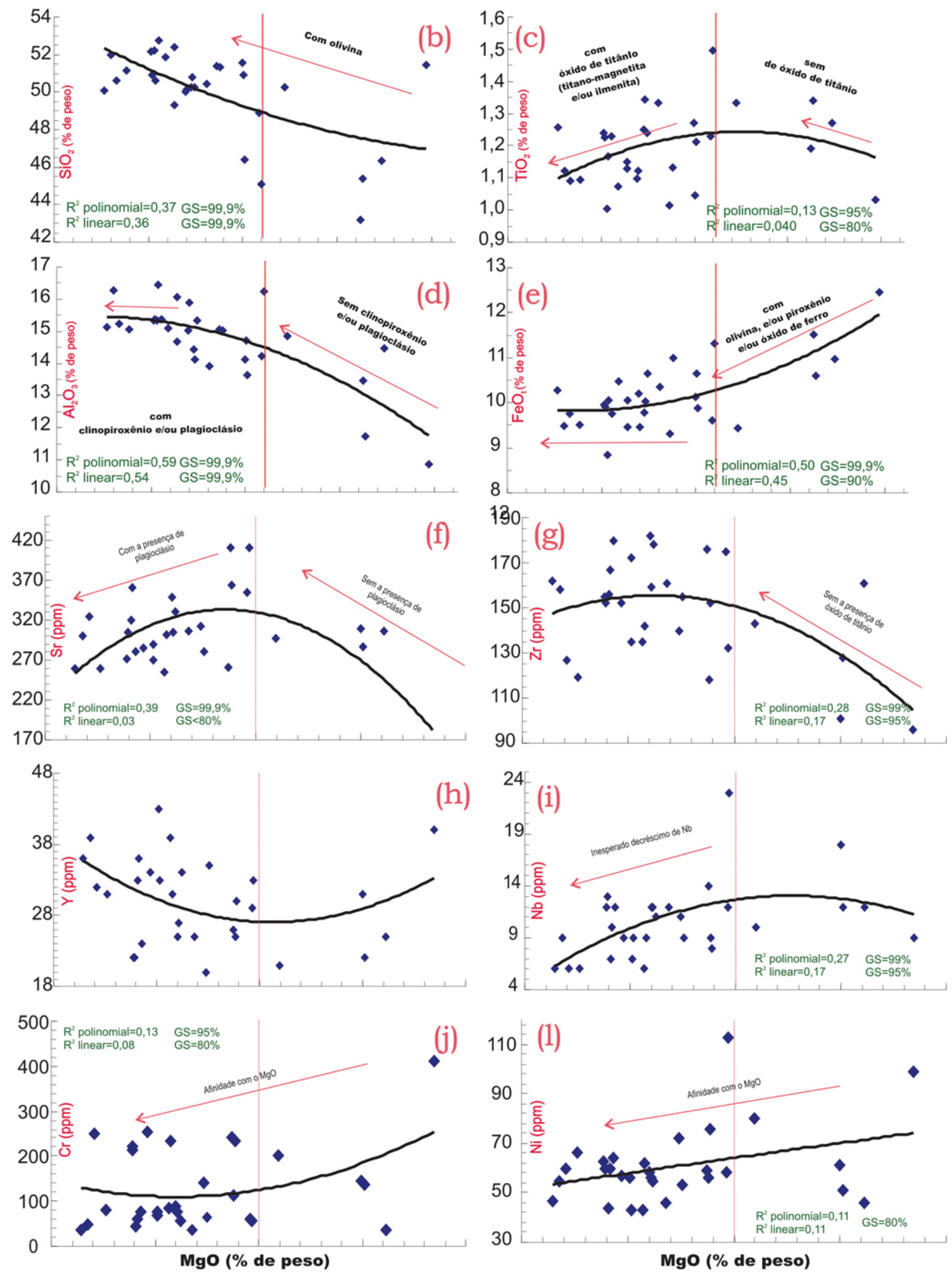

Figura 5 - Diagramas bivariantes mostrando a relação entre $\mathrm{MgO}$ e SiO2, TiO2, Al2O3 e FeOt (a, b, c, d) e $\mathrm{MgO}$ e $\mathrm{Sr}, \mathrm{Zr}, \mathrm{Y}, \mathrm{Nb}, \mathrm{Cr}$ e $\mathrm{Ni}(e, f, g, h, i, j)$. Os quadrados dos ceficientes de correlação lenar e polinomial (R2) e resppectivos graus de significância (GS, $n=30$ amostras e graus de liberdade $n-2)$, curvas polinomiais e possiveis fases fracionantes estão indicados. 
Tabela 4 - Comparação entre os valores de $R^{2}$ das funções lineares e polinomiais para os diagramas de variação dos elementos maiores e traços (os graus de significância das $R^{2}$ polinomiais para $n=30$ amostras e equações polinomiais de segunda ordem também são apresentadas).

\begin{tabular}{l|c|c|c|c}
\hline Óxido & $\mathbf{R}^{2}$ (linear) & $\mathbf{R}^{2}$ (polinomial) & $\begin{array}{c}\text { Graus de significância } \\
\text { polinomiais (GS) }\end{array}$ & $\begin{array}{c}\text { Equações polinomiais } \\
\text { de segunda ordem }\end{array}$ \\
\hline $\mathbf{S I O}_{2}$ & 0,36 & 0,37 & $99,9 \%$ & $\mathrm{y}=0,0675 \mathrm{x}^{2}-1,8653 \mathrm{x}+59,44$ \\
\hline $\mathbf{T I O}_{2}$ & 0,04 & 0,13 & $95,0 \%$ & $\mathrm{Y}=-0,0096 \mathrm{x}^{2}+0,1622 \mathrm{x}+0,555$ \\
\hline $\mathbf{A L}_{2} \mathbf{O}_{3}$ & 0,54 & 0,59 & $99,9 \%$ & $\mathrm{Y}=-0,0732 \mathrm{x}^{2}+0,6248 \mathrm{x}+14,128$ \\
\hline $\mathbf{F E O}_{\mathbf{T}}$ & 0,45 & 0,50 & $99,9 \%$ & $\mathrm{Y}=0,0515 \mathrm{x}^{2}-0,5092 \mathrm{x}+11,078$ \\
\hline $\mathbf{C A O}$ & 0,14 & 0,14 & $95,0 \%$ & $\mathrm{Y}=-0,0315 \mathrm{x}^{2}+0,1177 \mathrm{x}+8,4887$ \\
\hline $\mathbf{N A}_{2} \mathbf{O}$ & 0,09 & 0,13 & $95,0 \%$ & $\mathrm{Y}=-0,0345 \mathrm{x}^{2}+0,4351 \mathrm{x}+1,3865$ \\
\hline $\mathbf{K}_{\mathbf{2}} \mathbf{O}$ & 0,21 & 0,22 & $99,0 \%$ & $\mathrm{Y}=0,0362 \mathrm{x}^{2}-0,8261 \mathrm{x}+6,4501$ \\
\hline $\mathbf{P}_{2} \mathbf{O}_{\mathbf{5}}$ & 0,007 & 0,03 & $<80,0 \%$ & $\mathrm{Y}=-0,0021 \mathrm{x}^{2}+0,0328 \mathrm{x}+0,0544$ \\
\hline SR & 0,03 & 0,39 & $99,9 \%$ & $\mathrm{y}=-9,7105 \mathrm{x}^{2}+144,42 \mathrm{x}-203,78$ \\
\hline ZR & 0,17 & 0,28 & $99,0 \%$ & $\mathrm{y}=-2,1493 \mathrm{x}^{2}+27,957 \mathrm{x}+64,615$ \\
\hline $\mathbf{Y}$ & 0,006 & 0,15 & $95,0 \%$ & $\mathrm{y}=0,6355 \mathrm{x}^{2}-10,5 \mathrm{x}+70,407$ \\
\hline NB & 0,17 & 0,27 & $99,0 \%$ & $\mathrm{y}=-0,3339 \mathrm{x}^{2}+6,0546 \mathrm{x}-14,379$ \\
\hline $\mathbf{C R}$ & 0,08 & 0,13 & $95,0 \%$ & $\mathrm{y}=5,8998 \mathrm{x}^{2}-76,003 \mathrm{x}+354,86$ \\
\hline NI & 0,11 & 0,11 & $90,0 \%$ & $\mathrm{y}=-0,018 \mathrm{x}^{2}+3,3277 \mathrm{x}+38,641$ \\
\hline BA & 0,03 & 0,08 & $80,0 \%$ & $\mathrm{y}=-12,502 \mathrm{x}^{2}+173,84 \mathrm{x}+16,201$ \\
\hline RB & 0,13 & 0,14 & $95,0 \%$ & $\mathrm{y}=0,5324 \mathrm{x}^{2}-11,424 \mathrm{x}+89,087$ \\
\hline
\end{tabular}

\begin{tabular}{ccccc}
\hline Amostra & Simbolo & MgO (\%peso) & $($ La/Yb) & $($ La/Nb) \\
\hline RJS-156 & - & 7,88 & 5,78 & 0,97 \\
\hline RJS-92 6_3/13 & - & 4,57 & 5,88 & 5,30 \\
\hline
\end{tabular}

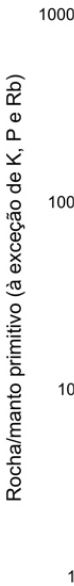

(a)

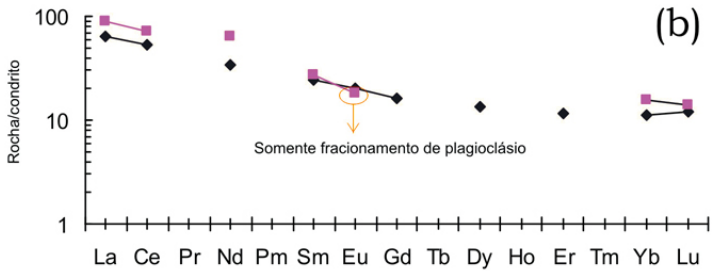

La Ce Pr Nd Pm Sm Eu Gd Tb Dy Ho Er Tm Yb Lu

b)

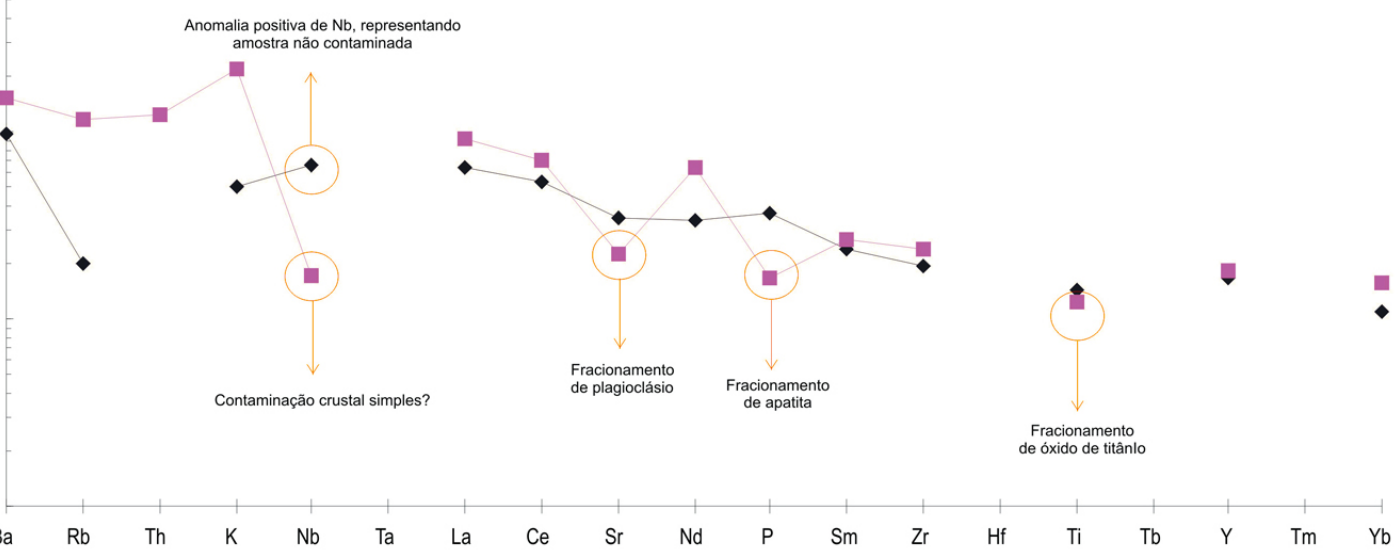

Figura 6-Análise da possivel evidência do processo de assimilação simples. a) Diagrama multielementar normalizado para o manto primitivo (Thompson et al. 1984; à exceção do K, P e Rb, que são valores de Sun 1980, e Ba =3,85 ppm de Hawkesworth et al. 1984); b) Diagrama de elementos terras raras normalizados para condrito Nakamura (1974).

Estas equações incluem os seguintes parâmetros: (1) Composição do contaminante $\left(\mathrm{C}_{\mathrm{a}}\right.$ para os elementos traços e $\varepsilon_{\mathrm{a}}$ para razões isotópicas); (2) Com- posição do magma parental não contaminado $\left(\mathrm{C}_{\mathrm{O}}\right.$ para os elementos traços e $\varepsilon_{O}$ para razões isotópicas); (3) Fração de massa do líquido residual (F); (4) O coefi- 
Tabela 5 - Razões $\mathrm{Zr} / \mathrm{Nb}$ e Y/Nb entre as amostras parental e mais evoluída dos basaltos do Cretáceo Inferior da bacia de Campos (RJS-156: $M g O=7,88 \%$ de peso; $R J S-92$ 6_3/13: $\mathrm{MgO}=4,57 \%$ de peso).

\begin{tabular}{l|l|c|l|c}
\hline \multicolumn{2}{c|}{ Amostra } & Zr/Nb & \multicolumn{1}{|c}{ Amostra } & Y/Nb \\
\hline Parental & RJS-156 & $\mathbf{5 , 7 3}$ & RJS-156 & $\mathbf{1 , 4 3}$ \\
\hline Derivativa & RJS-92 6_3/13 & $\mathbf{2 7 , 0 0}$ & RJS-92 6_3/13 & $\mathbf{6 , 0 0}$ \\
\hline \multicolumn{2}{c|}{ Fator de variação } & $\mathbf{5 , 7 0}$ & fator de variação & $\mathbf{4 , 1 9}$ \\
\hline
\end{tabular}

ciente de partição total cristal-líquido para os elementos traços durante o fracionamento (D = $\Sigma \% \mathrm{f}$. Kd); (5) A razão entre a quantidade de assimilação e a quantidade de cristalização fracionada (r).

Os valores destes parâmetros podem ser manipulados para explicar possíveis trends no diagrama de razões isotópicas $\left({ }^{87} \mathrm{Sr} /{ }^{86} \mathrm{Sr}_{130 \mathrm{Ma}}\right.$ versus $\left.{ }^{143} \mathrm{Nd} /{ }^{144} \mathrm{Nd}_{130 \mathrm{Ma}}\right)$. Para o parâmetro F, pode-se assumir valores do modelamento de elementos maiores, por exemplo. Os valores de $\mathrm{D}$ podem ser retirados da quantificação modal da assembléia de fenocristais realizada na petrografia. Os valores de $\mathrm{Kd}$ utilizados estão dispostos na literatura (Rollinson, 1993). Os valores de $\mathrm{r}$ não alcançam a unidade e podem ser manipulados para a obtenção dos trends de $A F C$. Segundo Taylor (1980), o valor de $\mathrm{r}$ para $1 \mathrm{~g}$ de rocha assimilada, a $150^{\circ} \mathrm{C}$, por um líquido com uma temperatura cerca de oito vezes maior (cerca de $1150^{\circ} \mathrm{C}$ ) equivaleria a $3,25 \mathrm{~g}$ de cristais fracionados $\mathrm{e}$, consequentemente, a um $\mathrm{r}=0,3(1 \mathrm{~g} / 3,25 \mathrm{~g})$. O parâmetro $\mathrm{r}$ está relacionado com o gradiente de temperatura existente entre o magma e a rocha contaminante; quanto menor o gradiente térmico, maiores os valores de r (DePaolo, 1981).

No modelamento quantitativo de $A F C$ para os basaltos de Campos, foram usados $\mathrm{D}_{\mathrm{Sr}}=1,21, \mathrm{D}_{\mathrm{Nd}}=0,15$ para uma assembléia de fenocristais composta por $5 \%$ de olivina, $30 \%$ de clinopiroxênio e $65 \%$ de plagioclásio extraída da análise modal quantificada na petrografia. Duas amostras da crosta superior e duas amostras representativas da crosta inferior locais. Granitóides e gnaisses (crosta superior; RJC-199 e RJC-200; Valente 1997) aflorantes no Rio de Janeiro, granulito (crosta inferior; MB-CM-43c; Valente 1997) de Juiz de Fora e ortognaisse (crosta inferior; RJ-15L; Fonceca 1993) da região de Cabo Frio (rochas aflorantes adjacente a bacia de Campos) foram utilizadas como possíveis contaminantes. Seus valores isotópicos e de elementos traços utilizados estão dispostos na tabela 6 . Foram utilizados um $\mathrm{r}=0,2$, para a possível solução de $A F C$ envolvendo crosta superior, e $\mathrm{r}=0,7$ para a crosta inferior.

Uma condição necessária para o modelamento de $A F C$ é a existência de uma amostra representativa de um líquido parental não contaminado. A amostra RJS-156, dentro da suíte basáltica de Campos possui

Tabela 6 - Valores das composições dos elementos traços e das razões isotópicas das amostras RJ/C-199 (granitóide, crosta superior), RJ/C-200 (gnaisse, crosta superior), MB-CM-43 (granulito, crosta inferior) (Valente 1997); RJ-15L (ortognaisse, crosta inferior) (Fonseca, 1993) e das amostras de Campos (Mizusaki et al. 1992) utilizadas no modelamento de AFC.

\begin{tabular}{|c|c|c|c|c|c|c|c|c|}
\hline & Amostra & $\begin{array}{l}\mathrm{RB} \\
(\mathrm{PPM})\end{array}$ & \begin{tabular}{|l}
$\mathrm{SR}$ \\
$(\mathrm{PPM})$
\end{tabular} & $\begin{array}{l}\mathrm{SM} \\
(\mathrm{PPM})\end{array}$ & $\begin{array}{l}\mathrm{ND} \\
(\mathrm{PPM})\end{array}$ & ${ }^{87} \mathrm{SR}^{86} \mathrm{SR}_{(130 \mathrm{MA})}$ & ${ }^{143} \mathrm{ND} /{ }^{144} \mathrm{ND}_{(130 \mathrm{MA})}$ & $\mathrm{RB} / \mathrm{SR}$ \\
\hline $\begin{array}{l}\text { CROSTA } \\
\text { SUPERIOR } \\
\text { (granitóide) }\end{array}$ & $\mathrm{RJ} / \mathrm{C}-199$ & 114 & 233 & 5 & 36 & 0,713704 & 0,511759 & 0,488 \\
\hline $\begin{array}{l}\text { CROSTA } \\
\text { SUPERIOR } \\
\text { (Gnaisse) }\end{array}$ & $\mathrm{RJ} / \mathrm{C}-200$ & 139 & 82 & 8 & 40 & 0,729307 & 0,511994 & 1,692 \\
\hline $\begin{array}{l}\text { CROSTA } \\
\text { INFERIOR } \\
\text { (granulito) } \\
\end{array}$ & MB-CM- $43 \mathrm{C}$ & 32 & 334 & 7 & 39 & 0,715804 & 0,511076 & 0,066 \\
\hline $\begin{array}{l}\text { CROSTA } \\
\text { INFERIOR } \\
\text { (ortognaisse) }\end{array}$ & RJ-15L & $X$ & $\mathrm{X}$ & $\mathrm{X}$ & $\mathrm{X}$ & 0,713699 & 0,510834 & 0,385 \\
\hline \multirow{7}{*}{$\begin{array}{l}\text { Basalto } \\
\text { de campos }\end{array}$} & $\mathrm{BD}-8$ & 36 & 272 & 5,42 & 23,97 & 0,708792 & 0,511464 & 0,132 \\
\hline & $\mathrm{BD}-4$ & 49 & 312 & 4,38 & 19,71 & 0,708610 & 0,511336 & 0,157 \\
\hline & RJS - 36 a & 6 & 411 & 3,71 & 13,12 & 0,709352 & 0,512155 & 0,0145 \\
\hline & $\mathrm{BD}-11 \mathrm{a}$ & 37 & 305 & 5,12 & 23,16 & 0,708771 & 0,511346 & 0,121 \\
\hline & RJS - 92a & 18 & 304 & 0 & 20 & 0,709073 & 0,511487 & 0,059 \\
\hline & RJS - 92b & 80 & 280 & 6,24 & 28,67 & 0,707832 & 0,511428 & 0,285 \\
\hline & RJS - 156 & 7 & 411 & 4,83 & 21,2 & 0,705769 & 0,512053 & 0,170 \\
\hline
\end{tabular}




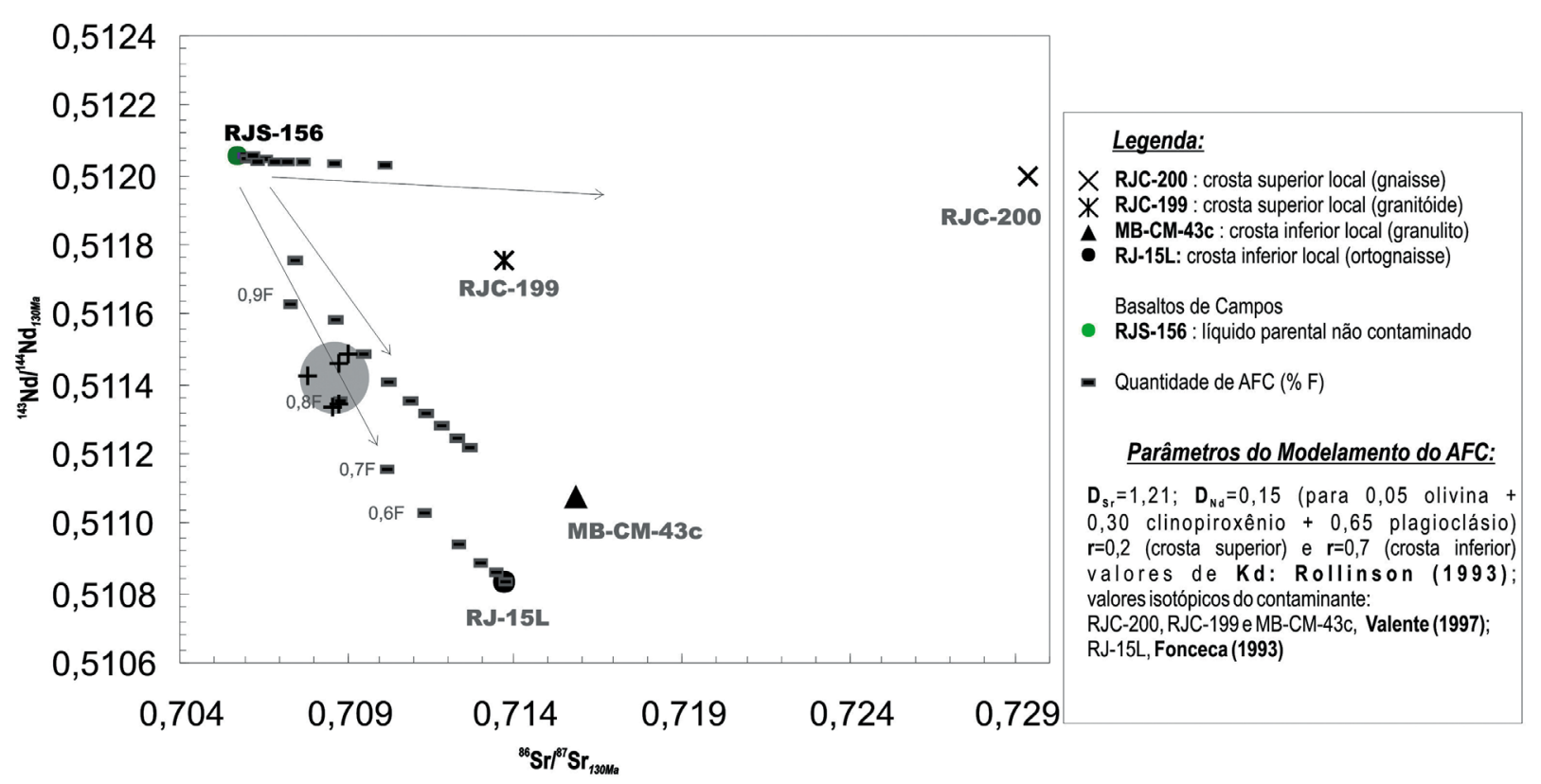

Figura 7 - Diagrama ${ }^{87} \mathrm{Sr}^{86} \mathrm{Sr}_{130 \mathrm{Ma}}$ versus ${ }^{143} \mathrm{Nd} /{ }^{144} \mathrm{Nd}{ }_{130 \mathrm{Ma}}$ com possíveis soluções de AFC para os basaltos de Campos.

composições de elementos traços que parecem satisfazer esta condição, por exemplo $\mathrm{La} / \mathrm{Nb}_{\mathrm{N}}=0,97 \mathrm{com}$ consequente anomalia positiva de $\mathrm{Nb}$ em diagrama multi-elementar (Fig. 6). Outra premissa é a de que a composição isotópica da suíte analisada possua valores aproximados, para quando forem plotadas no diagrama de variação isotópica, formem um grupo distinto dos contaminantes e da amostra parental não contaminada.

A figura 7 apresenta as possíveis soluções para o modelamento quantitativo do processo evolutivo de $A F C$ para os Basaltos de Campos. A melhor delas foi aquela que gerou um trend representando a assimilação de um contaminante da crosta inferior local (RJ-15L; Região de Cabo Frio, adjacente à bacia de Campos). Os valores do modelamento quantitativo de $A F C$ ficaram em cerca de $20 \%$, ou seja, correspondente a $0,80 \mathrm{~F}$.

CONCLUSÕES Modelamento geoquímico quantitativo demonstrou que os basaltos do Cretáceo Inferior da bacia de Campos evoluíram a partir de cerca de 30\% de $A F C$, envolvendo rochas ortognaissicas, representantes de crosta inferior, aflorantes na região de Cabo Frio, adjacente à bacia de Campos. A cristalização fracionada envolveu uma assembléia composta essencialmente por $5 \%$ de olivina, $30 \%$ de clinopiroxênio e $65 \%$ de plagioclásio. O envolvimento de crosta inferior local (possível localização da câmara magmática) no processo evolutivo dos basaltos de Campos encontra apoio na pronunciada anomalia negativa de $\mathrm{Nb}\left(\mathrm{La} / \mathrm{Nb}_{\mathrm{N}}\right.$ até $5,30)$ detectada principalmente nos seus membros mais evoluídos.

Agradecimentos Este trabalho é resultado parcial de pesquisas desenvolvidads durante a última década no CENPES/PETROBRAS. Janaina Lobo agradece: (1) as instituições PETROBRAS, FAPERJ, Faculdades de Geologia da UERJ e UFRRJ; (2) Aos gerentes João Cláudio Conceição, José Guimarães Rizzo e Edison José Milani pela gestão e apoio administrativo.

\section{Referências}

Allégre C.J. \& Minster J.F.. 1978. Quantitative models of trace element behaviour in magmatic process. Earth and Planetary Science Letters, 38:1-25.

Fonceca A.C. 1993. Esboço geocronológico da região de Cabo Frio, Estado do Rio de Janeiro. Tese de Doutorado, Instituto de Geociências, Universidade de São Paulo, $186 \mathrm{p}$.

Cox K.G., Bell J. D., Pankhurst R.J. 1979. The interpretation of igneous rocks. George Allen \& Unwin, London, 450pp.

DePaolo D.J. 1981. Trace element and isotopic effects of combined wallrock assimilation and fractional crystal- lisation. Earth and Planetary Science Letters, 53:189202.

Fodor R.V. \& Vetter S.K. 1984. Rift-zone magmatism: petrology of basaltic rocks transitional from CFB to MORB, Southeastern Brazil margin. Contributions to Mineralogy and Petrology, 88:307-321.

Fodor R.V. 1987. Low- and high- $\mathrm{TiO}_{2}$ flood basalts of southern Brazil: origin from picritic parentage and a common mantle source. Earth and Planetary Science Letters, 84:423-430.

Fodor R.V., McKee, E.H., Asmus H. E.. 1983/84. K-Ar ages and the opening of the South Atlantic ocean: ba- 
saltic rock from the Brazilian margin. Marine Geology, 54:M1-M8.

Hawkesworth C.J., Marsh J.S., Duncan A.R., Erlank A.J., Norry M.J. 1984. The role of continental lithosphere in the generation of the Karoo volcanic rocks: evidence from combined $\mathrm{Nd}$ - and $\mathrm{Sr}$-isotope studies. Special Publication of the Geological Society of South Africa, 13:341-354.

Hergt J.M., Peate D.W., Hawkesworth C.J. 1991. The petrogenesis of Mesozoic Gondwana low-Ti flood basalts. Earth and Planetary Science Letters, 105:134-148.

LeMaitre R.W. 1989. A classification of igneous rocks and glossary of terms. (Reccommendations of the International Union of Geological Sciences Subcommission on the Systematics of Igneous Rocks). Blackwell, Oxford, $193 \mathrm{p}$

Lobo J.T. 2000. Petrogênese dos basaltos do Cretáceo Inferior das bacias de Campos e Pelotas, SE do Brasil. Dissertação de Mestrado, Faculdade de Geologia, Universidade do Estado do Rio de Janeiro, 97p.

Lobo J.T., Valente S.C., Szatmari P., Duarte B.P. 2001b. Os basaltos do Cretáceo Inferior de Campos, Pelotas e províncias basálticas adjacentes: uma análise comparativa. In: VIII Congresso Brasileiro e Geoquímica e I Simpósio de Geoquímica dos Países do Mercosul, Curitiba. Anais, SBG, v.1, 100-101p.

Lobo J.T., Valente S.C., Szatmari P. 1999a. Diabásios da Serra do Mar e Basaltos da Bacia de Campos: comparação dos Processos de AFC através do modelamento geoquímico quantitativo. In: VI Simpósio de Geologia do Sudeste, São Paulo. Boletim de resumos, SBG-SP, 56p.

Lobo J. T., Valente S.C., Szatmari P., Duarte B.P. $2001 \mathrm{a}$. Campos, Pelotas e Paraná: comparação entre as suítes de alto- e baixo- $\mathrm{TiO}_{2}$. In: VIII Congresso Brasileiro de Geoquímica e I Simpósio de Geoquímica dos Países do Mercosul, Curitiba. Anais, SBGq, $100-101$ p.

Lobo J.T., Valente S.C., Thomaz Filho A., Szatmari P. 1999b. Modelamento geoquímico quantitativo dos processos evolutivos dos basaltos do Cretáceo Inferior da Bacia de Campos, SE do Brasil. In: V Congresso de Geoquímica dos Países de Lígua Portuguesa e VII Congresso Brasileiro de Geoquímica, Porto Seguro. Anais, SBG, 536- 538p.

Lobo J.T., Valente S.C., Szatmari P., Duarte B.P.. 2006. Tipos de fontes associadas às suítes basálticas de Campos e de Pelotas (Sul-Sudeste) e modelos geodinâmicos de ruptura do Gondwana ocidental. Boletim de Geociências da Petrobras, 12 (2):271-287.

Mackenzie W.S., Donaldson C.H., Guilford C. 1982. Atlas of igneous rocks and their textures. Harlow: Longman, 148pp.

Middlemost E.A.K. 1980. A contribuition to the nomenclature and classification of vocanic rocks. Geology Maga- zine, 117:51-57.

Mizusaki A.M.P., Petrini R., Bellieni G., Comin-Chiaramonti P., Dias J., DeMin A., Piccirillo E.M. 1992. Basalt magmatism along the passive continental margin of SE Brazil (Campos basin). Contributions to Mineralogy and Petrology, 111:143-160.

Mizusaki A.M.P., Thomaz Filho A., Valença J.G. 1988. Volcano-sedimentary sequence of Neocomian age in Campos basin (Brazil). Revista Brasileira de Geociências, 18:247-251.

Nakamura N.. 1974. Determination of REE, Ba, Fe, Mg, Na and $\mathrm{K}$ in carbonaceous and ordinary chondrites. Geochimica et Cosmochimica Acta, 38 (5):757-775.

Peate D.W., Hawkesworth C.J., Mantovani M.S.M. 1992. Chemical stratigraphy of the Paraná lavas (South America): classification of magma types and their spatial distribution. Bulletin of Volcanology, 55:119-139.

Rollinson H. 1993. Using geochemical data: evaluation, presentation and interpretation. Longman Scientific and Technical, 234pp.

Sun S.S. 1980. Lead isotopic study of young volcanic rocks from mid-ocean ridges, ocean islands and island arcs. Philosophical Transactions of the Royal Society of London, A297:409-445.

Taylor H. P. 1980. The effects of assimilation of country rocks by magmas on ${ }^{18} \mathrm{O} /{ }^{16} \mathrm{O}$ and ${ }^{87} \mathrm{Sr} /{ }^{86} \mathrm{Sr}$ systematics in igneous rocks. Earth and Planetary Science Letters, 47:243-254.

Taylor S.R. \& McLennan S.M. 1985. The continental crust: its composition and evolution. Blackwell \& Oxford. 312pp.

Thompson R.N. 1982. British Terciary volcanic province. Scotthish Journal Geology, 18:49-107.

Thompson R.N., Morrison M.A., Hendry, G.L., Parry S.J. 1984. An assessment of the relative roles of crust and mantle in magma genesis: an elemental approach. Philosophical Transactions of the Royal Society of London, A310:549-590.

Valente S.C. 1997. Geochemistry and isotopic constraints on the petrogenesis of the Cretaceous dykes of Rio de Janeiro, Brazil. Tese de doutoramento, Queen's University of Belfast, 400p.

Weaver B.L. \& Tarney J. 1981. Lewisian geochemistry and Archean crustal development models. Earth and Planetary Science Letters, 55:171-180.

Winchester J.A. \& Floyd P.A. 1977. Geochemical discrimination of different magma series and their differentiation products using immobile elements. Chemical Geology, 20:325-343.

Manuscrito AE-005/2006

Submetido em 06 janeiro de 2006 Aceito em 11 de maio de 2007 